Noname manuscript No.

(will be inserted by the editor)

\title{
Pointwise error estimate in difference setting for the two- dimensional nonlinear fractional complex Ginzburg-Landau equation
}

\author{
Qifeng Zhang • Jan S. Hesthaven · Zhi-zhong \\ Sun · Yunzhu Ren
}

Received: date / Accepted: date

\begin{abstract}
In this paper, we propose a three-level linearized implicit difference scheme for the two-dimensional spatial fractional nonlinear complex Ginzburg-Landau equation. We prove that the difference scheme is uniquely solvable, stable and convergent under mild conditions. The optimal convergence order $\mathcal{O}\left(\tau^{2}+h_{x}^{2}+h_{y}^{2}\right)$ is obtained in the pointwise sense by developing a new two-dimensional fractional Sobolev imbedding inequality based on the work in [K. Kirkpatrick, E. Lenzmann, G. Staffilani, Commun. Math. Phys., 317 (2013), pp. 563-591], an energy argument and careful attention to the nonlinear term. Numerical examples are presented to verify the validity of the theoretical results for different choices of the fractional orders $\alpha$ and $\beta$.
\end{abstract}

Keywords Fractional Ginzburg-Landau equation · Difference scheme · Pointwise error estimate $\cdot$ Stability · Convergence

Mathematics Subject Classification (2000) 65M06 $65 \mathrm{M} 12 \cdot 26 \mathrm{~A} 33 \cdot 35 \mathrm{R} 11$

Funding: The work of the first author was partially supported by Natural Science Foundation of China (Grant No. 11501514) and Visiting Scholar Program of China Scholarship Council (Grant No. 201908330528). The work of the third author was partially supported by the Natural Science Foundation of China (Grant No. 11671081).

Qifeng Zhang

Department of Mathematics, Zhejiang Sci-Tech University, Hangzhou, 310018, China

E-mail: zhangqifeng0504@gmail.com

Jan S. Hesthaven

SB-MATH-MCSS, École Polytechnique Fédérale de Lausanne (EPFL), 1015 Lausanne, Switzerland

E-mail: Jan.Hesthaven@epfl.ch

Zhi-zhong Sun

School of Mathematics, Southeast University, Nanjing, 210096, China

E-mail: zzsun@seu.edu.cn

Yunzhu Ren

Department of Mathematics, Zhejiang Sci-Tech University, Hangzhou, 310018, China

E-mail: 2669380451@qq.com 


\section{Introduction}

The Ginzburg-Landau equations have been extensively used as a model in fields including low-temperature superconductivity, superfluidity, Bose-Einstein condensation and physical phase transition processes [12,431. With the development of quantum mechanics, the concept of fractality has been successfully applied in fractal environments [11,36]. Laskin 1920] proposed fractional quantum mechanics by extending the Feynman path integral approach from the Brownian-like to the Lévy-like quantum mechanical paths. As a typical example, the space fractional complex Ginzburg-Landau equation was first discussed by Tarasov and Zaslavsky 32,33 , and involves a fractional spatial derivative instead of the Laplacian in the standard complex Ginzburg-Landau equation.

Much research has focused on investigations for the fractional complex Ginzburg-Landau equation from a theoretical view of point. For example, Guo and Huo [13] studied the local well-posedness in a subcritical space and the inviscid limit behavior of the solution of the fractional Ginzburg-Landau equation. Pu and Guo 27] considered the global well-posedness including global existence, some uniqueness criterion on weak solutions, and long-time dynamics for the nonlinear fractional complex Ginzburg-Landau equation. Next, Millot and Sire [25] considered the asymptotic analysis of the fractional complex Ginzburg-Landau equation in bounded domains. Bates et al. 23 discussed the initial boundary value problem of the fractional complex Ginzburg-Landau equation in three spatial dimensions, and derived an a priori estimate under the assumption that the nonlinearity satisfies certain growth conditions. In addition, there is work devoted to investigating soliton solutions [3] and random attractors of the fractional complex Ginzburg-Landau equation with additive noise 28 .

However, the theoretical analysis mostly offers qualitative results, which are insufficient for quantitative calculations since the analytical solutions of the nonlinear fractional complex Ginzburg-Landau equations is generally unavailable. Thus, efficient and stable numerical algorithms are important and necessary. For example, combined with a compact difference technique, Wang and Huang proposed a fourth-order linearized compact scheme for the fractional Ginzburg-Landau equation [34]. Later, Li and Huang [21,22] constructed efficient difference schemes for the nonlinear fractional Ginzburg-Landau equation and its coupled case based on the Galerkin finite element method. He and Pan [15] considered an implicit finite difference scheme for the one-dimensional fractional Ginzburg-Landau equation, for which unconditional stability and convergence in the maximum norm was proved. Zhang et al. 38 proposed a fast iterative solver based on a new splitting technique for the fractional Ginzburg-Landau equation, and Hao and Sun 14 analyzed a linearized compact scheme for the one-dimensional complex fractional Ginzburg-Landau equation, and established convergence in a pointwise sense.

To the authors' knowledge, there is limited work in the literature which focuses on the numerical solution of the nonlinear fractional complex Ginzburg-Landau equations in high dimensions. Recently, Zhang et al. [39] utilized linearized ADI schemes to solve the two-dimensional space-fractional nonlinear Ginzburg-Landau equation, and the order of the convergence was proved to be $\mathcal{O}\left(\tau^{2}+h_{x}^{2}+h_{y}^{2}\right)$, where $\tau$ denotes the temporal step size and $h_{x}$, $h_{y}$ the spatial step sizes in $x$-direction and $y$-direction, respectively. However, a very strong stability condition is required. Subsequently, an efficient three-level finite difference method with a preconditioning technique to solve the two-dimensional case with convergence of $\mathcal{O}\left(\tau^{2}+h_{x}^{2}+h_{y}^{2}\right)$ was proposed, greatly decreasing the CPU time [40]. In addition, there is work 
concerned with the numerical solution of the two-dimensional space-fractional nonlinear Ginzburg-Landau equation by using Fourier spectral methods [24, 26].

Although a significant amount of relevant works have already been developed, they often lack theoretical support, or only offer error estimates in $L^{2}$. In fact, $L^{2}$-error estimates do not provide insight into the local numerical error as the time evolves. However, error estimates in the pointwise sense are grid-independent. Therefore, pointwise error estimates are relevant in theoretical analysis and practical application. Although there is already an embedding theorem [17. for the fractional Riesz derivative in one dimension, it is unfortunately not applicable to the two-dimensional case, and it is required to develop new embedding theorems for the two-dimensional case.

In this paper, we analyze the pointwise error of an efficient numerical algorithm for the two-dimensional fractional Ginzburg-Landau equation in space as

$$
\begin{aligned}
& \partial_{t} u-(\nu+\mathbf{i} \eta)\left(\partial_{x}^{\alpha}+\partial_{y}^{\beta}\right) u+(\kappa+\mathbf{i} \zeta)|u|^{2} u-\gamma u=0, \quad(x, y) \in \mathbb{R}^{2}, 0<t \leqslant T, \\
& u(x, y, 0)=\varphi(x, y), \quad(x, y) \in \mathbb{R}^{2} .
\end{aligned}
$$

Here $\mathbf{i}=\sqrt{-1}$ denotes the imaginary unit, $\partial_{x}^{\alpha}$ and $\partial_{x}^{\beta}$ in (1.1) denote the Riesz fractional derivative operators for $1<\alpha, \beta \leqslant 2$ and are defined as [12],

$$
\partial_{x}^{\alpha} u(x, y, t)=-\frac{1}{2 \cos (\alpha \pi / 2) \Gamma(2-\alpha)} \frac{\partial^{2}}{\partial x^{2}} \int_{-\infty}^{\infty}|x-\xi|^{1-\alpha} u(\xi, y, t) \mathrm{d} \xi
$$

and

$$
\partial_{y}^{\beta} u(x, y, t)=-\frac{1}{2 \cos (\beta \pi / 2) \Gamma(2-\beta)} \frac{\partial^{2}}{\partial y^{2}} \int_{-\infty}^{\infty}|y-\varpi|^{1-\beta} u(x, \varpi, t) \mathrm{d} \varpi .
$$

For $\alpha=\beta=2$, the Riesz fractional derivative operator recovers the classical Laplacian and (1.1) -(1.2) reduces to the classical complex Ginzburg-Landau equation, which have been extensively studied numerically, see e.g., [5, 8,10, 18, 30]. When $1<\alpha, \beta \leqslant 2$ and $\nu, \kappa \rightarrow 0$, the solution of the fractional Ginzburg-Landau equation asymptotically approximates the fractional Schrödinger equation for which, a three-level linearized scheme and $L^{2}$-norm error estimates were presented [41.

The main contributions of the present paper are as follows:

- We develop a fractional Sobolev inequality at a discrete level. This is useful for the pointwise error estimate of two-dimensional spatial fractional problems.

- Pointwise error estimate is proved rigorously for the numerical scheme of the twodimensional nonlinear fractional Ginzburg-Landau equation.

- Solution uniqueness and stability of the numerical scheme are ensured under very mild conditions.

- The use of the difference scheme is illustrated through some examples of the nonlinear complex fractional Ginzburg-Landau equation.

The remainder of the paper is organized as follows. In Section 2 we provide some preliminary material including the definition of fractional Sobolev norms, the discrete Sobolev inequality, and discuss the equivalence of the fractional seminorm. Section 3 is the main part, where a three-level linearized finite difference scheme is proposed and the corresponding theoretical analysis including uniqueness, convergence in the pointwise sense, and stability are presented. Numerical support for the theoretical analysis is shown in Section 4 followed by the concluding remarks in Section 5. The derivation of the local truncation errors is provided in the Appendix. 


\section{Preliminary}

In this section, we introduce the fractional Sobolev norms and the discrete Sobolev inequality, which play important roles in the following analysis.

\subsection{Fractional Sobolev norms}

Denote $Z_{h}$ as the infinite grid with grid points $x_{i}=i h_{x}, y_{j}=j h_{y}$ for $(i, j) \in Z$. For any grid functions $u=\left\{u_{i j}\right\}, v=\left\{v_{i j}\right\}$ on $Z_{h}$, we define the discrete inner product and the corresponding $l^{2}$-norm as

$$
(u, v)=h_{x} h_{y} \sum_{i, j \in Z} u_{i j} v_{i j}^{*}, \quad\|u\|^{2}=(u, u), \quad\|u\|_{l^{\infty}}=\max _{i, j \in Z}\left|u_{i j}\right|,
$$

where $v_{i j}^{*}$ denotes the complex conjugate of $v_{i j}$. Set $L_{h}^{2}:=\left\{u \mid u=\left\{u_{i j}\right\},\|u\|^{2}<+\infty\right\}$. For $u \in L_{h}^{2}\left(\left[-\pi / h_{x}, \pi / h_{x}\right] \times\left[-\pi / h_{y}, \pi / h_{y}\right]\right)$, the semi-discrete Fourier transform $\hat{u}$ in two dimension is defined as

$$
\hat{u}\left(k_{1}, k_{2}\right):=\frac{h_{x} h_{y}}{(\sqrt{2 \pi})^{2}} \sum_{i \in Z} \sum_{j \in Z} u_{i j} \exp \left(-\mathbf{i} k_{1} x_{i}-\mathbf{i} k_{2} y_{j}\right) .
$$

The continuous inversion formula is given as

$$
u_{i j}=\frac{1}{2 \pi} \int_{-\frac{\pi}{h_{y}}}^{\frac{\pi}{h_{y}}} \int_{-\frac{\pi}{h_{x}}}^{\frac{\pi}{h_{x}}} \hat{u}\left(k_{1}, k_{2}\right) \exp \left(\mathbf{i} k_{1} x_{i}+\mathbf{i} k_{2} y_{j}\right) \mathrm{d} k_{1} \mathrm{~d} k_{2},
$$

and we define the inner product

$$
(u, v)=\int_{-\frac{\pi}{h_{y}}}^{\frac{\pi}{h_{y}}} \int_{-\frac{\pi}{h_{x}}}^{\frac{\pi}{h_{x}}} \hat{u}\left(k_{1}, k_{2}\right) \hat{v}^{*}\left(k_{1}, k_{2}\right) \mathrm{d} k_{1} \mathrm{~d} k_{2} .
$$

Therefore,

$$
\|u\|^{2}=\int_{-\frac{\pi}{h_{y}}}^{\frac{\pi}{h_{y}}} \int_{-\frac{\pi}{h_{x}}}^{\frac{\pi}{h_{x}}}\left|\hat{u}\left(k_{1}, k_{2}\right)\right|^{2} \mathrm{~d} k_{1} \mathrm{~d} k_{2} .
$$

Note that (2.1) and (2.3) are equivalent due to (2.2). For given constants $\alpha, \beta>1$, we define the fractional Sobolev norm $\|\cdot\|_{H_{h}^{\alpha, \beta}}$, semi-norms $|\cdot|_{H_{h}^{\alpha, \beta}}$ and $|\cdot|_{H_{h}^{\frac{\alpha}{2}}, \frac{\beta}{2}}$ as

$$
\begin{aligned}
&\|u\|_{H_{h}^{\alpha, \beta}}^{2}=\int_{-\frac{\pi}{h_{y}}}^{\frac{\pi}{h_{y}}} \int_{-\frac{\pi}{h_{x}}}^{\frac{\pi}{h_{x}}}\left(1+\left|k_{1}\right|^{\alpha}+\left|k_{2}\right|^{\beta}+2\left|k_{1}\right|^{\alpha}\left|k_{2}\right|^{\beta}+\left|k_{1}\right|^{2 \alpha}+\left|k_{2}\right|^{2 \beta}\right)\left|\hat{u}\left(k_{1}, k_{2}\right)\right|^{2} \mathrm{~d} k_{1} \mathrm{~d} k_{2}, \\
&|u|_{H_{h}^{\frac{\alpha}{2}, \frac{\beta}{2}}}^{2}=\int_{-\frac{\pi}{h_{y}}}^{\frac{\pi}{h_{y}}} \int_{-\frac{\pi}{h_{x}}}^{\frac{\pi}{h_{x}}}\left(\left|k_{1}\right|^{\alpha}+\left|k_{2}\right|^{\beta}\right)\left|\hat{u}\left(k_{1}, k_{2}\right)\right|^{2} \mathrm{~d} k_{1} \mathrm{~d} k_{2}, \\
&|u|_{H_{h}^{\alpha, \beta}}^{2}=\int_{-\frac{\pi}{h_{y}}}^{\frac{\pi}{h_{y}}} \int_{-\frac{\pi}{h_{x}}}^{\frac{\pi}{h_{x}}}\left(2\left|k_{1}\right|^{\alpha}\left|k_{2}\right|^{\beta}+\left|k_{1}\right|^{2 \alpha}+\left|k_{2}\right|^{2 \beta}\right)\left|\hat{u}\left(k_{1}, k_{2}\right)\right|^{2} \mathrm{~d} k_{1} \mathrm{~d} k_{2} .
\end{aligned}
$$


Clearly,

$$
\|u\|_{H_{h}^{\alpha, \beta}}^{2}=\|u\|^{2}+|u|_{H_{h}^{\frac{\alpha}{2}, \frac{\beta}{2}}}^{2}+|u|_{H_{h}^{\alpha, \beta}}^{2} .
$$

We define $H_{h}^{\alpha, \beta}:=\left\{u \mid u=\left\{u_{i j}\right\},\|u\|_{H_{h}^{\alpha, \beta}}<+\infty\right\}$.

\subsection{Discrete Sobolev inequality}

Since the standard discrete Sobolev inequality only holds for one-dimensional problems (see Lemma 3.1 in [17]), we introduce a new discrete Sobolev inequality for the two-dimensional case.

Lemma 2.1 (Discrete Sobolev inequality) For every $\alpha, \beta>1$ and $u \in H_{h}^{\alpha, \beta}$, it holds that

$$
\|u\|_{l \infty} \leqslant C_{\alpha, \beta}\|u\|_{H_{h}^{\alpha, \beta}} .
$$

where $C_{\alpha, \beta}>0$ is a constant depending on $\alpha, \beta$ but is independent of $h_{x}$ and $h_{y}$.

Proof We have

$$
\begin{aligned}
\left|u_{i j}\right|= & \left|\frac{1}{2 \pi} \int_{-\frac{\pi}{h_{y}}}^{\frac{\pi}{h_{y}}} \int_{-\frac{\pi}{h_{x}}}^{\frac{\pi}{h_{x}}} \hat{u}\left(k_{1}, k_{2}\right) e^{\mathrm{i} k_{1} x_{i}+\mathbf{i} k_{2} x_{j}} \mathrm{~d} k_{1} \mathrm{~d} k_{2}\right| \\
\leqslant & \frac{1}{2 \pi} \int_{-\frac{\pi}{h_{y}}}^{\frac{\pi}{h_{y}}} \int_{-\frac{\pi}{h_{x}}}^{\frac{\pi}{h_{x}}}\left|\hat{u}\left(k_{1}, k_{2}\right)\right| \mathrm{d} k_{1} \mathrm{~d} k_{2} \\
= & \frac{1}{2 \pi} \int_{-\frac{\pi}{h_{y}}}^{\frac{\pi}{h_{y}}} \int_{-\frac{\pi}{h_{x}}}^{\frac{\pi}{h_{x}}}\left(1+\left|k_{1}\right|^{\alpha}\right)^{-\frac{1}{2}}\left(1+\left|k_{2}\right|^{\beta}\right)^{-\frac{1}{2}}\left|\hat{u}\left(k_{1}, k_{2}\right)\right|\left(1+\left|k_{1}\right|^{\alpha}\right)^{\frac{1}{2}}\left(1+\left|k_{2}\right|^{\beta}\right)^{\frac{1}{2}} \mathrm{~d} k_{1} \mathrm{~d} k_{2} \\
\leqslant & \frac{1}{2 \pi}\left(\int_{-\frac{\pi}{h_{y}}}^{\frac{\pi}{h_{y}}} \int_{-\frac{\pi}{h_{x}}}^{\frac{\pi}{h_{x}}} \frac{1}{\left(1+\left|k_{1}\right|^{\alpha}\right)\left(1+\left|k_{2}\right|^{\beta}\right)} \mathrm{d} k_{1} \mathrm{~d} k_{2}\right)^{\frac{1}{2}} \\
& \times\left(\int_{-\frac{\pi}{h_{y}}}^{\frac{\pi}{h_{y}}} \int_{-\frac{\pi}{h_{x}}}^{\frac{\pi}{h_{x}}}\left(1+\left|k_{1}\right|^{\alpha}\right)\left(1+\left|k_{2}\right|^{\beta}\right)\left|\hat{u}\left(k_{1}, k_{2}\right)\right|^{2} \mathrm{~d} k_{1} \mathrm{~d} k_{2}\right)^{\frac{1}{2}} \\
\leqslant & C_{\alpha, \beta}\|u\|_{H_{h}^{\alpha, \beta}},
\end{aligned}
$$

where $C_{\alpha, \beta}=\frac{1}{2 \pi}\left(\int_{-\frac{\pi}{h_{y}}}^{\frac{\pi}{h_{y}}} \int_{-\frac{\pi}{h_{x}}}^{\frac{\pi}{h_{x}}} \frac{1}{\left(1+\left|k_{1}\right|^{\alpha}\right)\left(1+\left|k_{2}\right|^{\beta}\right)} \mathrm{d} k_{1} \mathrm{~d} k_{2}\right)^{\frac{1}{2}}$ is bounded provided $\alpha, \beta>$ 1.

Define

$\mathscr{C}^{n+\alpha, n+\beta}\left(\mathbb{R}^{2}\right)=\left\{f\left|\int_{\mathbb{R}^{2}}\left(1+\left|\varpi_{1}\right|^{n+\alpha}+\left|\varpi_{2}\right|^{n+\beta}\right)\right| \hat{f}\left(\varpi_{1}, \varpi_{2}\right) \mid \mathrm{d} \varpi_{1} \mathrm{~d} \varpi_{2}<\infty, f \in L^{1}\left(\mathbb{R}^{2}\right)\right\}$. 
Lemma 2.2 (see [6]) For every $\alpha, \beta>1$, suppose $f(x, y) \in \mathscr{C}^{2+\alpha, 2+\beta}\left(\mathbb{R}^{2}\right)$ and the fractional central difference is defined as

$$
\delta_{x}^{\alpha} f(x, y)=-h_{x}^{-\alpha} \sum_{p=-\infty}^{+\infty} g_{p}^{(\alpha)} f\left(x-p h_{x}, y\right), \quad \delta_{y}^{\beta} f(x, y)=-h_{y}^{-\beta} \sum_{q=-\infty}^{+\infty} g_{q}^{(\beta)} f\left(x, y-q h_{y}\right) .
$$

Then

$$
\delta_{x}^{\alpha} f(x, y)=\partial_{x}^{\alpha} f(x, y)+\mathcal{O}\left(h_{x}^{2}\right), \quad \delta_{y}^{\beta} f(x, y)=\partial_{y}^{\beta} f(x, y)+\mathcal{O}\left(h_{y}^{2}\right) .
$$

Provided $g_{k}^{(s)}(s=\alpha$ or $\beta)$ is defined as

$$
g_{k}^{(s)}=\frac{(-1)^{k} \Gamma(s+1)}{\Gamma(s / 2-k+1) \Gamma(s / 2+k+1)}, \quad k \in \mathbb{Z} .
$$

Lemma 2.3 (Equivalence of the fractional seminorm) For every $\alpha, \beta>1$ and $u \in H_{h}^{\alpha, \beta}$, the second-order fractional centeral difference operator for the space fractional derivatives is defined as

$$
\delta_{x}^{\alpha} u_{i j}:=-\frac{1}{h_{x}^{\alpha}} \sum_{l=-\infty}^{\infty} g_{l}^{(\alpha)} u_{i-l, j}, \quad \delta_{y}^{\beta} u_{i j}:=-\frac{1}{h_{y}^{\beta}} \sum_{m=-\infty}^{\infty} g_{m}^{(\beta)} u_{i, j-m} .
$$

Then

$$
\widetilde{C}_{\alpha, \beta}|u|_{H_{h}^{\frac{\alpha}{2}, \frac{\beta}{2}}}^{2} \leqslant\left(-\left(\delta_{x}^{\alpha}+\delta_{y}^{\beta}\right) u, u\right) \leqslant|u|_{H_{h}^{\frac{\alpha}{2}, \frac{\beta}{2}}}^{2},
$$

and

$$
\left(\widetilde{C}_{\alpha, \beta}\right)^{2}|u|_{H_{h}^{\alpha, \beta}}^{2} \leqslant\left(\left(\delta_{x}^{\alpha}+\delta_{y}^{\beta}\right) u,\left(\delta_{x}^{\alpha}+\delta_{y}^{\beta}\right) u\right) \leqslant|u|_{H_{h}^{\alpha, \beta}}^{2},
$$

where $\widetilde{C}_{\alpha, \beta}=(2 \backslash \pi)^{\max \{\alpha, \beta\}}$.

Proof In [6], the generating function for $z \in \mathbb{R}$ is given by

$$
|2 \sin (z / 2)|^{\alpha}=\sum_{l=-\infty}^{\infty} g_{l}^{\alpha} \exp (\mathbf{i} l z)
$$

Taking the semi-discrete Fourier transform of $\left(\delta_{x}^{\alpha}+\delta_{y}^{\beta}\right) u_{i j}$, and noting (2.14), we have

$$
\begin{aligned}
\left(\delta_{x}^{\alpha} \widehat{+\delta_{y}^{\beta}}\right) u_{i j}= & -\frac{1}{h_{x}^{\alpha}} \sum_{l=-\infty}^{\infty} g_{l}^{(\alpha)} \hat{u}_{i-l, j}-\frac{1}{h_{y}^{\beta}} \sum_{m=-\infty}^{\infty} g_{m}^{(\beta)} \hat{u}_{i, j-m} \\
= & -\left(\frac{1}{h_{x}^{\alpha}} \sum_{l=-\infty}^{\infty} g_{l}^{(\alpha)} \frac{h_{x} h_{y}}{2 \pi} \sum_{i \in Z} \sum_{j \in Z} u_{i j} \exp \left(-\mathbf{i} k_{1}(i-l) h_{x}-\mathbf{i} k_{2} y_{j}\right)\right. \\
& \left.+\frac{1}{h_{y}^{\beta}} \sum_{m=-\infty}^{\infty} g_{m}^{(\beta)} \frac{h_{x} h_{y}}{2 \pi} \sum_{i \in Z} \sum_{j \in Z} u_{i j} \exp \left(-\mathbf{i} k_{1} x_{i}-\mathbf{i} k_{2}(j-m) h_{y}\right)\right)
\end{aligned}
$$




$$
\begin{aligned}
& =-\left(\frac{1}{h_{x}^{\alpha}} \sum_{l=-\infty}^{\infty} g_{l}^{(\alpha)} \exp \left(\mathbf{i} l k_{1} h_{x}\right)+\frac{1}{h_{y}^{\beta}} \sum_{m=-\infty}^{\infty} g_{m}^{(\beta)} \exp \left(\mathbf{i} m k_{2} h_{y}\right)\right) \hat{u}\left(k_{1}, k_{2}\right) \\
& =-\left(\frac{1}{h_{x}^{\alpha}}\left|2 \sin \left(\frac{k_{1} h_{x}}{2}\right)\right|^{\alpha}+\frac{1}{h_{y}^{\beta}}\left|2 \sin \left(\frac{k_{2} h_{y}}{2}\right)\right|^{\beta}\right) \hat{u}\left(k_{1}, k_{2}\right) .
\end{aligned}
$$

Using Parseval's identity, we have

$$
\begin{aligned}
& \left(-\left(\delta_{x}^{\alpha}+\delta_{y}^{\beta}\right) u, u\right) \\
= & \int_{-\frac{\pi}{h_{y}}}^{\frac{\pi}{h_{y}}} \int_{-\frac{\pi}{h_{x}}}^{\frac{\pi}{h_{x}}}\left(\frac{1}{h_{x}^{\alpha}}\left|2 \sin \left(\frac{k_{1} h_{x}}{2}\right)\right|^{\alpha}+\frac{1}{h_{y}^{\beta}}\left|2 \sin \left(\frac{k_{2} h_{y}}{2}\right)\right|^{\beta}\right)\left|\hat{u}\left(k_{1}, k_{2}\right)\right|^{2} \mathrm{~d} k_{1} \mathrm{~d} k_{2} .
\end{aligned}
$$

Since $k_{1} \in\left[-\pi / h_{x}, \pi / h_{x}\right]$ and $k_{2} \in\left[-\pi / h_{y}, \pi / h_{y}\right]$, we have $k_{1} h_{x} / 2 \in[-\pi / 2, \pi / 2]$ and $k_{2} h_{y} / 2 \in[-\pi / 2, \pi / 2]$. As $(2 / \pi) \cdot|x| \leqslant|\sin (x)| \leqslant|x|, x \in[-\pi / 2, \pi / 2]$, it yields

$$
\frac{2}{\pi}\left|\frac{k_{1} h_{x}}{2}\right| \leqslant\left|\sin \left(\frac{k_{1} h_{x}}{2}\right)\right| \leqslant\left|\frac{k_{1} h_{x}}{2}\right|, \quad \frac{2}{\pi}\left|\frac{k_{2} h_{y}}{2}\right| \leqslant\left|\sin \left(\frac{k_{2} h_{y}}{2}\right)\right| \leqslant\left|\frac{k_{2} h_{y}}{2}\right|
$$

and

$$
\left(\frac{2}{\pi}\right)^{\alpha}\left|k_{1}\right|^{\alpha} \leqslant \frac{1}{h_{x}^{\alpha}}\left|2 \sin \left(\frac{k_{1} h_{x}}{2}\right)\right|^{\alpha} \leqslant\left|k_{1}\right|^{\alpha}, \quad\left(\frac{2}{\pi}\right)^{\beta}\left|k_{2}\right|^{\beta} \leqslant \frac{1}{h_{y}^{\beta}}\left|2 \sin \left(\frac{k_{2} h_{y}}{2}\right)\right|^{\beta} \leqslant\left|k_{2}\right|^{\beta} .
$$

Hence, we recover

$$
\begin{gathered}
\widetilde{C}_{\alpha, \beta} \int_{-\frac{\pi}{h_{y}}}^{\frac{\pi}{h_{y}}} \int_{-\frac{\pi}{h_{x}}}^{\frac{\pi}{h_{x}}}\left(\left|k_{1}\right|^{\alpha}+\left|k_{2}\right|^{\beta}\right)\left|\hat{u}\left(k_{1}, k_{2}\right)\right|^{2} \mathrm{~d} k_{1} \mathrm{~d} k_{2} \\
\leqslant\left(-\left(\delta_{x}^{\alpha}+\delta_{y}^{\beta}\right) u, u\right) \leqslant \int_{-\frac{\pi}{h_{y}}}^{\frac{\pi}{h_{y}}} \int_{-\frac{\pi}{h_{x}}}^{\frac{\pi}{h_{x}}}\left(\left|k_{1}\right|^{\alpha}+\left|k_{2}\right|^{\beta}\right)\left|\hat{u}\left(k_{1}, k_{2}\right)\right|^{2} \mathrm{~d} k_{1} \mathrm{~d} k_{2},
\end{gathered}
$$

or,

$$
\widetilde{C}_{\alpha, \beta}|u|_{H_{h}^{\frac{\alpha}{2}, \frac{\beta}{2}}}^{2} \leqslant\left(-\left(\delta_{x}^{\alpha}+\delta_{y}^{\beta}\right) u, u\right) \leqslant|u|_{H_{h}^{\frac{\alpha}{2}, \frac{\beta}{2}}}^{2} .
$$

Following the same approach, we obtain

$$
\begin{aligned}
& \left(\left(\delta_{x}^{\alpha}+\delta_{y}^{\beta}\right) u,\left(\delta_{x}^{\alpha}+\delta_{y}^{\beta}\right) u\right) \\
= & \int_{-\frac{\pi}{h_{y}}}^{\frac{\pi}{h_{y}}} \int_{-\frac{\pi}{h_{x}}}^{\frac{\pi}{h_{x}}}\left(\frac{1}{h_{x}^{\alpha}}\left|2 \sin \left(\frac{k_{1} h_{x}}{2}\right)\right|^{\alpha}+\frac{1}{h_{y}^{\beta}}\left|2 \sin \left(\frac{k_{2} h_{y}}{2}\right)\right|^{\beta}\right)^{2}\left|\hat{u}\left(k_{1}, k_{2}\right)\right|^{2} \mathrm{~d} k_{1} \mathrm{~d} k_{2},
\end{aligned}
$$

to recover

$$
\begin{gathered}
\left(\widetilde{C}_{\alpha, \beta}\right)^{2} \int_{-\frac{\pi}{h_{y}}}^{\frac{\pi}{h_{y}}} \int_{-\frac{\pi}{h_{x}}}^{\frac{\pi}{h_{x}}}\left(\left|k_{1}\right|^{\alpha}+\left|k_{2}\right|^{\beta}\right)^{2}\left|\hat{u}\left(k_{1}, k_{2}\right)\right|^{2} \mathrm{~d} k_{1} \mathrm{~d} k_{2} \\
\leqslant\left(\left(\delta_{x}^{\alpha}+\delta_{y}^{\beta}\right) u,\left(\delta_{x}^{\alpha}+\delta_{y}^{\beta}\right) u\right) \leqslant \int_{-\frac{\pi}{h_{y}}}^{\frac{\pi}{h_{y}}} \int_{-\frac{\pi}{h_{x}}}^{\frac{\pi}{h_{x}}}\left(\left|k_{1}\right|^{\alpha}+\left|k_{2}\right|^{\beta}\right)^{2}\left|\hat{u}\left(k_{1}, k_{2}\right)\right|^{2} \mathrm{~d} k_{1} \mathrm{~d} k_{2} .
\end{gathered}
$$

Hence,

$$
\left(\widetilde{C}_{\alpha, \beta}|u|_{H_{h}^{\alpha, \beta}}^{2}\right)^{2} \leqslant\left(\left(\delta_{x}^{\alpha}+\delta_{y}^{\beta}\right) u,\left(\delta_{x}^{\alpha}+\delta_{y}^{\beta}\right) u\right) \leqslant|u|_{H_{h}^{\alpha, \beta}}^{2} .
$$

This completes the proof. 
Remark 2.1 Denote $\Omega=[a, b] \times[c, d]$ and suppose $f^{*}(x, y)$ is defined by

$$
f^{*}(x, y)= \begin{cases}f(x, y) & (x, y) \in \Omega, \\ 0, & (x, y) \in \mathbb{R}^{2} \backslash \Omega,\end{cases}
$$

such that $f^{*}(x, y) \in \mathscr{C}^{2+\alpha, 2+\beta}\left(\mathbb{R}^{2}\right)$. It follows from Lemma 2.2 that for $1<\alpha, \beta \leqslant 2$,

$\left(\partial_{x}^{\alpha}+\partial_{y}^{\beta}\right) f=-h_{x}^{-\alpha} \sum_{i=-\left[\frac{b-x}{h_{x}}\right]}^{\left[\frac{x-a}{h_{x}}\right]} g_{p}^{\alpha} f\left(x-i h_{x}, y\right)-h_{y}^{-\beta} \sum_{j=-\left[\frac{d-y}{h_{y}}\right]}^{\left[\frac{y-c}{h_{y}}\right]} g_{q}^{\beta} f\left(x, y-j h_{y}\right)+\mathcal{O}\left(h_{x}^{2}+h_{y}^{2}\right)$.

Denote

$$
\left(\delta_{x}^{\alpha}+\delta_{y}^{\beta}\right) f=-h_{x}^{-\alpha} \sum_{i=-\left[\frac{b-x}{h_{x}}\right]}^{\left[\frac{x-a}{h_{x}}\right]} g_{p}^{\alpha} f\left(x-i h_{x}, y\right)-h_{y}^{-\beta} \sum_{j=-\left[\frac{d-y}{h_{y}}\right]}^{\left[\frac{y-c}{h_{y}}\right]} g_{q}^{\beta} f\left(x, y-j h_{y}\right) .
$$

\section{A three-level linearized scheme and the numerical analysis}

We assume that the solution decays to zero smoothly in space. For the finite difference method to capture the behavior of the solution, we set the function values outside the domain $\Omega$ to zero without losing accuracy. The fractional Ginzburg Landau equation (1.1)(1.2) can be truncated on the domain $\Omega$, subject to homogeneous boundary condition as

$$
\begin{array}{ll}
\partial_{t} u-(\nu+\mathbf{i} \eta)\left(\partial_{x}^{\alpha}+\partial_{y}^{\beta}\right) u+(\kappa+\mathbf{i} \zeta)|u|^{2} u-\gamma u=0, & (x, y) \in \Omega, 0<t \leqslant T, \\
u(x, y, 0)=\varphi(x, y), & (x, y) \in \mathbb{R}^{2}, \\
u(x, y, t)=0, & (x, y) \in \mathbb{R}^{2} \backslash \Omega, 0<t \leqslant T,
\end{array}
$$

where $1<\alpha, \beta \leqslant 2, \nu>0, \kappa>0, \eta, \zeta, \gamma$ are real constants, $\varphi(x, y)$ is a given function vanishing in $\mathbb{R}^{2} \backslash \Omega$. The boundary condition (3.3) is referred to as the nonlocal volume constraint and the corresponding problem (1.1)-(1.2) can be viewed as the volume constraint problem [7,9]. The fractional derivatives (1.3) and (1.4) are reduced to

$$
\partial_{x}^{\alpha} u(x, y, t)=-\frac{1}{2 \cos (\alpha \pi / 2) \Gamma(2-\alpha)} \frac{\partial^{2}}{\partial x^{2}} \int_{a}^{b}|x-\xi|^{1-\alpha} u(\xi, y, t) \mathrm{d} \xi
$$

and

$$
\partial_{y}^{\beta} u(x, y, t)=-\frac{1}{2 \cos (\beta \pi / 2) \Gamma(2-\beta)} \frac{\partial^{2}}{\partial y^{2}} \int_{c}^{d}|y-\varpi|^{1-\beta} u(x, \varpi, t) \mathrm{d} \varpi .
$$

Given a positive integer $N$, let $t^{n}=n \tau, 0 \leqslant n \leqslant N$, where $\tau=T / N$. Denote $\Omega_{\tau}=\left\{t^{n} \mid 0 \leqslant\right.$ $n \leqslant N\}$ and define a grid function $v=\left\{v^{n} \mid 0 \leqslant n \leqslant N\right\}$ on $\Omega_{\tau}$. Denote

$$
\begin{aligned}
& v^{\bar{n}}=\frac{1}{2}\left(v^{n+1}+v^{n-1}\right), \quad \Delta_{t} v^{n}=\frac{1}{2 \tau}\left(v^{n+1}-v^{n-1}\right), \\
& \delta_{t} v^{n+\frac{1}{2}}=\frac{1}{\tau}\left(v^{n+1}-v^{n}\right), \quad v^{\hat{n}}=\frac{1}{4}\left(v^{n+2}+2 v^{n}+v^{n-2}\right) .
\end{aligned}
$$


For two positive integers $M_{x}, M_{y}$, let $h_{x}=(b-a) / M_{x}, h_{y}=(d-c) / M_{y}$, and denote $x_{i}=a+i h_{x}, y_{j}=c+j h_{y}, 0 \leqslant i \leqslant M_{x}, 0 \leqslant j \leqslant M_{y}$. Denote $\bar{\Omega}_{h}=\left\{\left(x_{i}, y_{j}\right) \mid 0 \leqslant i \leqslant\right.$ $\left.M_{x} 0 \leqslant j \leqslant M_{y}\right\}, \quad \Omega_{h}=\bar{\Omega}_{h} \bigcap \Omega, \partial \Omega_{h}=\bar{\Omega}_{h} \bigcap \partial \Omega, \bar{\omega}=\left\{(i, j) \mid 0 \leqslant i \leqslant M_{x}, 0 \leqslant j \leqslant M_{y}\right\}$, $\omega=\left\{(i, j) \mid\left(x_{i}, y_{j}\right) \in \Omega_{h}\right\}, \partial \omega=\bar{\omega} \backslash \omega$.

\subsection{Some useful lemmas}

Define $\mathcal{V}_{h}=\left\{v \mid v=\left\{v_{i j}\right\}, 0 \leqslant i \leqslant M_{x}, 0 \leqslant j \leqslant M_{y}\right\}, \stackrel{\circ}{\mathcal{V}}_{h}=\left\{v \in \mathcal{V}_{h}, v_{i j}=0\right.$ if $\left.(i, j) \in \partial \omega\right\}$. We have the following lemmas.

Lemma 3.4 [16] For any grid functions $u, v \in \dot{\mathcal{V}}_{h}$, it holds that

$$
\Delta_{t}\left(u^{n} v^{n}\right)=u^{\bar{n}} \Delta_{t} v^{n}+v^{\bar{n}} \Delta_{t} u^{n} .
$$

Lemma 3.5 35. For any grid functions $u, v \in \dot{\mathcal{V}}_{h}$, there exists a linear operator $\Lambda^{\alpha, \beta}$ such that

$$
\left(-\left(\delta_{x}^{\alpha}+\delta_{y}^{\beta}\right) u, v\right)=\left(\Lambda^{\alpha, \beta} u, \Lambda^{\alpha, \beta} v\right) .
$$

Lemma 3.6 (Discrete Gronwall's inequality, 29]) Suppose $\left\{E^{k}\right\}_{n=0}^{\infty}$ is a nonnegative sequence which satisfies

$$
E^{n+1} \leqslant(1+\sigma \tau) E^{n}+\tau g, \quad n=0,1,2, \cdots .
$$

Then we have

$$
E^{n} \leqslant \exp (\sigma n \tau)\left(E^{0}+\frac{g}{\sigma}\right), \quad n=1,2, \cdots,
$$

where $\sigma$ and $g$ are nonnegative constants.

3.2 The derivation of the linearized difference scheme

Assume there exists a solution $u(x, y, t) \in C^{4,4,3}(\Omega \times[0, T])$ for (3.1)-(3.3), define

$$
u^{*}(x, y)= \begin{cases}u(x, y, t), & (x, y) \in \Omega, \\ 0, & (x, y) \in R^{2} \backslash \Omega,\end{cases}
$$

and suppose $u^{*} \in \mathscr{C}^{2+\alpha, 2+\beta}\left(\mathbb{R}^{2}\right)$. Define the grid function

$$
U_{i j}^{n}=u\left(x_{i}, y_{j}, t^{n}\right), \quad(i, j) \in \bar{\omega}, 0 \leqslant n \leqslant N .
$$

Now, we consider (3.1) at the point $\left(\mathbf{x}_{i j}, t^{n}\right)$

$$
\begin{aligned}
& \partial_{t} u\left(x_{i}, y_{j}, t^{n}\right)-(\nu+\mathbf{i} \eta)\left(\partial_{x}^{\alpha}+\partial_{y}^{\beta}\right) u\left(x_{i}, y_{j}, t^{n}\right)+(\kappa+\mathbf{i} \zeta)\left|u\left(x_{i}, y_{j}, t_{n}\right)\right|^{2} u\left(x_{i}, y_{j}, t^{n}\right) \\
& \quad-\gamma u\left(x_{i}, y_{j}, t^{n}\right)=0, \quad(i, j) \in \omega, 0 \leqslant n \leqslant N .
\end{aligned}
$$

Using the Taylor expansion, we have

$$
\begin{aligned}
& \partial_{t} u\left(x_{i}, y_{j}, t^{n}\right)=\Delta_{t} U_{i j}^{n}+\mathcal{O}\left(\tau^{2}\right), \\
& \left|u\left(x_{i}, y_{j}, t^{n}\right)\right|^{2} u\left(x_{i}, y_{j}, t^{n}\right)=\left|U_{i j}^{n}\right|^{2} U_{i j}^{\bar{n}}+\mathcal{O}\left(\tau^{2}\right), \\
& u\left(x_{i}, y_{j}, t^{n}\right)=U_{i j}^{\bar{n}}+\mathcal{O}\left(\tau^{2}\right) .
\end{aligned}
$$


From Lemma 2.2 and 2.15), we have

$$
\begin{aligned}
\left(\partial_{x}^{\alpha}+\partial_{y}^{\beta}\right) u\left(x_{i}, y_{j}, t^{n}\right) & =\left(\partial_{x}^{\alpha}+\partial_{y}^{\beta}\right) U_{i j}^{\bar{n}}+\mathcal{O}\left(\tau^{2}\right) \\
& =-\frac{1}{h_{x}^{\alpha}} \sum_{l=0}^{M_{x}} g_{i-l}^{(\alpha)} U_{l j}^{\bar{n}}-\frac{1}{h_{y}^{\beta}} \sum_{m=0}^{M_{y}} g_{j-m}^{(\beta)} U_{i m}^{\bar{n}}+\mathcal{O}\left(\tau^{2}+h_{x}^{2}+h_{y}^{2}\right) \\
& =\left(\delta_{x}^{\alpha}+\delta_{y}^{\beta}\right) U_{i j}^{\bar{n}}+\mathcal{O}\left(\tau^{2}+h_{x}^{2}+h_{y}^{2}\right) .
\end{aligned}
$$

Substituting (3.6)-(3.9) into (3.5) gives

$$
\begin{gathered}
\Delta_{t} U_{i j}^{n}-(\nu+\mathbf{i} \eta)\left(\delta_{x}^{\alpha}+\delta_{y}^{\beta}\right) U_{i j}^{\bar{n}}+(\kappa+\mathbf{i} \zeta)\left|U_{i j}^{n}\right|^{2} U_{i j}^{\bar{n}}-\gamma U_{i j}^{\bar{n}}=R_{i j}^{n}, \\
(i, j) \in \omega, 1 \leqslant n \leqslant N-1 .
\end{gathered}
$$

Therefore, there exists a positive constant $C_{R}$ such that

$$
\begin{aligned}
& \left|R_{i j}^{n}\right| \leqslant C_{R}\left(\tau^{2}+h_{x}^{2}+h_{y}^{2}\right), \quad(i, j) \in \omega, 1 \leqslant n \leqslant N-1, \\
& \left|\Delta_{t} R_{i j}^{n}\right| \leqslant C_{R}\left(\tau^{2}+h_{x}^{2}+h_{y}^{2}\right), \quad(i, j) \in \omega, 2 \leqslant n \leqslant N-1 .
\end{aligned}
$$

The detailed proofs of (3.11) and (3.12) are technical, and postponed to the Appendix.

For the approximation scheme at the first level, we consider (3.1) at $\left(x_{i}, y_{j}, t^{\frac{1}{2}}\right)$ and have

$$
\begin{aligned}
& \partial_{t} u\left(x_{i}, y_{j}, t^{\frac{1}{2}}\right)-(\nu+\mathbf{i} \eta)\left(\delta_{x}^{\alpha}+\delta_{y}^{\beta}\right) u\left(x_{i}, y_{j}, t^{\frac{1}{2}}\right)+(\kappa+\mathbf{i} \zeta)\left|u\left(x_{i}, y_{j}, t^{\frac{1}{2}}\right)\right|^{2} u\left(x_{i}, y_{j}, t^{\frac{1}{2}}\right) \\
& -\gamma u\left(x_{i}, y_{j}, t^{\frac{1}{2}}\right)=0, \quad(i, j) \in \omega .
\end{aligned}
$$

From the Taylor expansion, we have

$$
u\left(x_{i}, y_{j}, t^{\frac{1}{2}}\right)=u\left(x_{i}, y_{j}, t_{0}\right)+\frac{\tau}{2} \partial_{t} u\left(x_{i}, y_{j}, t_{0}\right)+\mathcal{O}\left(\tau^{2}\right) .
$$

Using 3.5, we have

$$
\begin{aligned}
\partial_{t} u\left(x_{i}, y_{j}, t_{0}\right)= & (\nu+\mathbf{i} \eta)\left(\partial_{x}^{\alpha}+\partial_{y}^{\beta}\right) u\left(x_{i}, y_{j}, t_{0}\right)-(\kappa+\mathbf{i} \zeta)\left|u\left(x_{i}, y_{j}, t_{0}\right)\right|^{2} u\left(x_{i}, y_{j}, t_{0}\right) \\
& +\gamma u\left(x_{i}, y_{j}, t_{0}\right), \quad(i, j) \in \omega .
\end{aligned}
$$

By the linearized Crank-Nicholson method, we obtain

$$
\delta_{t} U_{i j}^{\frac{1}{2}}-(\nu+\mathbf{i} \eta)\left(\delta_{x}^{\alpha}+\delta_{y}^{\beta}\right) U_{i j}^{\frac{1}{2}}+(\kappa+\mathbf{i} \zeta)\left|\hat{u}_{i j}^{0}\right|^{2} U_{i j}^{\frac{1}{2}}-\gamma U_{i j}^{\frac{1}{2}}=R_{i j}^{0}, \quad(i, j) \in \omega,
$$

where

$$
\hat{u}_{i j}^{0}=U_{i j}^{0}+\frac{\tau}{2}\left((\nu+\mathbf{i} \eta)\left(\partial_{x}^{\alpha}+\partial_{y}^{\beta}\right) U_{i j}^{0}-(\kappa+\mathbf{i} \zeta)\left|U_{i j}^{0}\right|^{2} U_{i j}^{0}+\gamma U_{i j}^{0}\right)
$$

and

$$
\left|R_{i j}^{0}\right| \leqslant C_{R}\left(\tau^{2}+h_{x}^{2}+h_{y}^{2}\right), \quad(i, j) \in \omega .
$$

Noticing the initial and boundary conditions (3.2) and (3.3), we have

$$
\begin{aligned}
& U_{i j}^{0}=\varphi\left(x_{i}, y_{j}\right), \quad(i, j) \in \bar{\omega}, \\
& U_{i j}^{n}=0, \quad(i, j) \in \partial \omega, 0 \leqslant n \leqslant N .
\end{aligned}
$$


Ignoring the higher order terms $R_{i j}^{n}$ in (3.10) and $R_{i j}^{0}$ in (3.14) and replacing the exact solution $U_{i j}^{n}$ with the numerical solution $u_{i j}^{n}$, the finite difference scheme becomes

$$
\begin{aligned}
& \delta_{t} u_{i j}^{\frac{1}{2}}-(\nu+\mathbf{i} \eta)\left(\delta_{x}^{\alpha}+\delta_{y}^{\beta}\right) u_{i j}^{\frac{1}{2}}+(\kappa+\mathbf{i} \zeta)\left|\hat{u}_{i j}^{0}\right|^{2} u_{i j}^{\frac{1}{2}}-\gamma u_{i j}^{\frac{1}{2}}=0, \quad(i, j) \in \omega, \\
& \Delta_{t} u_{i j}^{n}-(\nu+\mathbf{i} \eta)\left(\delta_{x}^{\alpha}+\delta_{y}^{\beta}\right) u_{i j}^{\bar{n}}+(\kappa+\mathbf{i} \zeta)\left|u_{i j}^{n}\right|^{2} u_{i j}^{\bar{n}}-\gamma u_{i j}^{\bar{n}}=0, \\
& \quad(i, j) \in \omega, 1 \leqslant n \leqslant N-1, \\
& u_{i j}^{0}=\varphi\left(x_{i}, y_{j}\right), \quad(i, j) \in \bar{\omega}, \\
& u_{i j}^{n}=0, \quad(i, j) \in \partial \omega, 0 \leqslant n \leqslant N .
\end{aligned}
$$

\subsection{Unique solvability}

Theorem 3.1 (Uniqueness) When $\gamma \leqslant 0$ or $\gamma>0$ and $\tau<\frac{1}{\gamma}$, the linearized finite difference scheme (3.18)-(3.21) is uniquely solvable.

Proof It is easy to see that $u^{0}$ is determined by (3.20) and (3.21). The linear system of equations for $u^{1}$ is defined in (3.18) and (3.21). Considering the homogeneous linear system of equations, we have

$$
\begin{aligned}
\frac{1}{\tau} u_{i j}^{1}-\frac{1}{2}(\nu+\mathbf{i} \eta)\left(\delta_{x}^{\alpha}+\delta_{y}^{\beta}\right) u_{i j}^{1}+\frac{1}{2}(\kappa+\mathbf{i} \zeta)\left|\hat{u}_{i j}^{0}\right|^{2} u_{i j}^{1}-\frac{\gamma}{2} u_{i j}^{1}=0, & (i, j) \in \omega, \\
u_{i j}^{1}=0, & (i, j) \in \partial \omega .
\end{aligned}
$$

Taking the discrete inner product of (3.22) with $u^{1}$, we have

$$
\frac{1}{\tau}\left\|u^{1}\right\|^{2}-\frac{1}{2}(\nu+\mathbf{i} \eta)\left(\left(\delta_{x}^{\alpha}+\delta_{y}^{\beta}\right) u^{1}, u^{1}\right)+\frac{1}{2}(\kappa+\mathbf{i} \zeta)\left(\left|\hat{u}_{i j}^{0}\right|^{2} u^{1}, u^{1}\right)-\frac{\gamma}{2}\left\|u^{1}\right\|^{2}=0 .
$$

By Lemma 3.5. we have

$$
\operatorname{Re}\left(-\left(\delta_{x}^{\alpha}+\delta_{y}^{\beta}\right) u^{1}, u^{1}\right)=\left\|\Lambda^{\alpha, \beta} u^{1}\right\|^{2} .
$$

Taking the real part in (3.24), we have

$$
\frac{1}{\tau}\left\|u^{1}\right\|^{2}+\frac{\nu}{2}\left\|\Lambda^{\alpha, \beta} u^{1}\right\|^{2}+\frac{\kappa}{2}\left(\left|u_{i j}^{0}\right|^{2} u^{1}, u^{1}\right)-\frac{\gamma}{2}\left\|u^{1}\right\|^{2}=0 .
$$

When $\gamma \leqslant 0$ or $\gamma>0$ and $\tau<\frac{2}{\gamma}$, we have $\left\|u^{1}\right\|=0$, and hence the difference scheme (3.18) is uniquely solvable.

For given $u^{n}, u^{n-1}$, (3.19) and (3.21) also determine a linear system of equations. Considering its homogeneous linear system of equations, we have

$$
\begin{aligned}
& \frac{1}{2 \tau} u_{i j}^{n+1}-\frac{1}{2}(\nu+\mathbf{i} \eta)\left(\delta_{x}^{\alpha}+\delta_{y}^{\beta}\right) u_{i j}^{n+1}+\frac{1}{2}(\kappa+\mathbf{i} \zeta)\left|u_{i j}^{n}\right|^{2} u_{i j}^{n+1}-\frac{\gamma}{2} u_{i j}^{n+1}=0, \quad(i, j) \in \omega, \\
& u_{i j}^{n+1}=0, \quad(i, j) \in \partial \omega, 1 \leqslant n \leqslant N-1 .
\end{aligned}
$$

Similar to the case of $n=1$, we have

$$
\frac{1}{2 \tau}\left\|u^{n+1}\right\|^{2}+\frac{\nu}{2}\left\|\Lambda^{\alpha, \beta} u^{n+1}\right\|^{2}+\frac{\kappa}{2}\left(\left|u^{n}\right|^{2} u^{n+1}, u^{n+1}\right)-\frac{\gamma}{2}\left\|u^{n+1}\right\|^{2}=0 .
$$

When $\gamma \leqslant 0$ or $\gamma>0$ and $\tau<\frac{1}{\gamma}$, we have $\left\|u^{n+1}\right\|=0$ and hence $u^{n+1}$ is uniquely solvable. Using induction, this completes the proof. 
3.4 Convergence

Denote the error

$$
e_{i j}^{n}=U_{i j}^{n}-u_{i j}^{n}, \quad(i, j) \in \bar{\omega}, 0 \leqslant n \leqslant N,
$$

and

$|\Omega|=(b-a)(d-c), \quad\|u\|_{\infty}=\sup _{(x, y) \in \Omega, t \in[0, T]}\{|u(x, y, t)|\}, \quad C_{u}=\max \left\{\|u\|_{\infty},\left\|u_{t}\right\|_{\infty}\right\}$,

$c_{1}=\frac{1}{2} \sqrt{\kappa^{2}+\zeta^{2}}\left[C_{u}\left(2 C_{u}+1\right)+\left(C_{u}+1\right)^{2}\right]+|\gamma|+\frac{1}{2}, \quad c_{2}=\frac{1}{16 c_{1}^{2}}+\frac{|\Omega| C_{R}^{2}}{4 c_{1}}$,

$c_{3}=\exp \left(16 c_{1} T\right) c_{2}, \quad c_{4}=\max \left\{c_{1}, \frac{|\gamma|^{2}}{8 \nu}\right\}, \quad c_{5}=16 c_{1} c_{3}+4 C_{R}^{2}|\Omega| T$,

$c_{6}=\frac{1}{4 \nu^{2}}(34+4 T) C_{R}^{2}, \quad c_{7}=\left(C_{u}^{2}+2 C_{u}\right)\left(\sqrt{\kappa^{2}+\zeta^{2}}+\frac{|\gamma|}{2}+1\right), \quad c_{8}=2\left(C_{u}+1\right)^{2}$,

$c_{9}=\frac{c_{7}}{\nu} \sqrt{\kappa^{2}+\zeta^{2}}, \quad c_{10}=c_{7} \max \left\{\frac{40 \sqrt{\kappa^{2}+\zeta^{2}}}{\nu} T, \frac{34\left(\kappa^{2}+\zeta^{2}\right)}{\nu}\right\}$,

$c_{11}=\left[\left(\frac{4}{\nu^{2}}+\frac{4}{|\gamma| \nu}\right) C_{R}^{2}|\Omega|+4\left(c_{5}+c_{6}+c_{7}\right)\right] \exp \left(\left(160 \widetilde{C}+64 c_{4}+16\right) T\right)$,

$c_{12}=\frac{C_{\alpha, \beta}}{\widetilde{C}_{\alpha, \beta}} \sqrt{c_{3}+c_{11}}$.

We have the following convergence result.

Theorem 3.2 (Convergence) Let $u(x, y, t)$ be the solution of the problem (3.1)-(3.3), $\left\{u_{i j}^{n} \mid(i, j) \in \bar{\omega}, 0 \leqslant n \leqslant N\right\}$ be the solution of the scheme 3.18)-3.21. We have

$$
\left\|e^{n}\right\|_{l^{\infty}} \leqslant c_{12}\left(\tau^{2}+h_{x}^{2}+h_{y}^{2}\right), \quad 0 \leqslant n \leqslant N .
$$

Proof Subtracting (3.18)-3.21) from (3.10), (3.14), (3.16) and (3.17), we obtain the error equations

$$
\begin{aligned}
& \delta_{t} e_{i j}^{\frac{1}{2}}-(\nu+\mathbf{i} \eta)\left(\delta_{x}^{\alpha}+\delta_{y}^{\beta}\right) e_{i j}^{\frac{1}{2}}+(\kappa+\mathbf{i} \zeta)\left|\hat{u}_{i j}^{0}\right|^{2} e_{i j}^{\frac{1}{2}}-\gamma e_{i j}^{\frac{1}{2}}=R_{i j}^{0}, \quad(i, j) \in \omega, \\
& \Delta_{t} e_{i j}^{n}-(\nu+\mathbf{i} \eta)\left(\delta_{x}^{\alpha}+\delta_{y}^{\beta}\right) e_{i j}^{\bar{n}}+(\kappa+\mathbf{i} \zeta) p_{i j}^{n}-\gamma e_{i j}^{\bar{n}}=R_{i j}^{n}, \\
& \quad(i, j) \in \omega, 1 \leqslant n \leqslant N-1, \\
& e_{i j}^{0}=0, \quad(i, j) \in \bar{\omega}, \\
& e_{i j}^{n}=0, \quad(i, j) \in \partial \omega, 0 \leqslant n \leqslant N,
\end{aligned}
$$

where

$$
p_{i j}^{n}=\left|U_{i j}^{n}\right|^{2} U_{i j}^{\bar{n}}-\left|u_{i j}^{n}\right|^{2} u_{i j}^{\bar{n}}=\left|u_{i j}^{n}\right|^{2} e_{i j}^{\bar{n}}+U_{i j}^{n} U_{i j}^{\bar{n}}\left(e_{i j}^{n}\right)^{*}+U_{i j}^{\bar{n}}\left(u_{i j}^{n}\right)^{*} e_{i j}^{n} .
$$

We will prove (3.28) by induction. By employing (3.31), it's easy to see that (3.28) holds for $k=0$. When $k=1$, taking the discrete inner product of (3.29) with $e^{1}$, we have

$$
\frac{1}{\tau}\left\|e^{1}\right\|^{2}-\frac{1}{2}(\nu+\mathbf{i} \eta)\left(\left(\delta_{x}^{\alpha}+\delta_{y}^{\beta}\right) e^{1}, e^{1}\right)+\frac{1}{2}(\kappa+\mathbf{i} \zeta)\left(\left|\hat{u}^{0}\right|^{2} e^{1}, e^{1}\right)-\frac{\gamma}{2}\left\|e^{1}\right\|^{2}=\left(R^{0}, e^{1}\right) .
$$


Considering the real part of (3.34), we have

$$
\frac{1}{\tau}\left\|e^{1}\right\|^{2}+\frac{\nu}{2}\left\|\Lambda^{\alpha, \beta} e^{1}\right\|^{2}+\frac{\kappa}{2}\left(\left|\hat{u}^{0}\right|^{2} e^{1}, e^{1}\right)-\frac{\gamma}{2}\left\|e^{1}\right\|^{2}=\operatorname{Re}\left(R^{0}, e^{1}\right),
$$

and

$$
\frac{1}{\tau}\left\|e^{1}\right\|^{2}+\frac{\nu}{2}\left\|\Lambda^{\alpha, \beta} e^{1}\right\|^{2} \leqslant \frac{|\gamma|}{2}\left\|e^{1}\right\|^{2}+\left\|R^{0}\right\| \cdot\left\|e^{1}\right\| .
$$

Hence, when $\tau \leqslant \frac{1}{|\gamma|}$, we have

$$
\left\|e^{1}\right\| \leqslant \frac{\tau}{1-\frac{|\gamma| \tau}{2}}\left\|R^{0}\right\| \leqslant 2 \tau\left\|R^{0}\right\| .
$$

By using (3.36) and (3.37), we also obtain

$$
\frac{\tau \nu}{2}\left\|\Lambda^{\alpha, \beta} e^{1}\right\|^{2} \leqslant \frac{|\gamma| \tau}{2}\left\|e^{1}\right\|^{2}+\tau\left\|R^{0}\right\| \cdot\left\|e^{1}\right\|
$$

To recover

$$
\left\|\Lambda^{\alpha, \beta} e^{1}\right\| \leqslant \frac{2}{\sqrt{|\gamma| \nu}}\left\|R^{0}\right\| .
$$

For further estimation of $e^{1}$, take the discrete inner product of (3.29) with $-\left(\delta_{x}^{\alpha}+\delta_{y}^{\beta}\right) e^{1}$ as

$$
\begin{aligned}
& \frac{1}{\tau}\left\|\Lambda^{\alpha, \beta} e^{1}\right\|^{2}+\frac{1}{2}(\nu+\mathbf{i} \eta)\left\|\left(\delta_{x}^{\alpha}+\delta_{y}^{\beta}\right) e^{1}\right\|^{2}+\frac{1}{2}(\kappa+\mathbf{i} \zeta)\left(\left|\hat{u}^{0}\right|^{2} \Lambda^{\alpha, \beta} e^{1}, \Lambda^{\alpha, \beta} e^{1}\right)-\frac{\gamma}{2}\left\|\Lambda^{\alpha, \beta} e^{1}\right\|^{2} \\
& \quad=-\left(R^{0},\left(\delta_{x}^{\alpha}+\delta_{y}^{\beta}\right) e^{1}\right) .
\end{aligned}
$$

Taking the real part of (3.40), we have

$$
\left.\left(\frac{1}{\tau}-\frac{\gamma}{2}\right)\left\|\Lambda^{\alpha, \beta} e^{1}\right\|^{2}+\frac{\nu}{2}\left\|\left(\delta_{x}^{\alpha}+\delta_{y}^{\beta}\right) e^{1}\right\|^{2}+\frac{\kappa}{2}\left(\left|\hat{u}^{0}\right|^{2} \Lambda^{\alpha, \beta} e^{1}, \Lambda^{\alpha, \beta} e^{1}\right) \leqslant\left\|R^{0}\right\| \cdot \|\left(\delta_{x}^{\alpha}+\delta_{y}^{\beta}\right) e^{1}\right) \| .
$$

When $\gamma \leqslant 0$ or $\gamma>0$ and $\tau \leqslant \frac{2}{\gamma}$, we have

$$
\left\|\left(\delta_{x}^{\alpha}+\delta_{y}^{\beta}\right) e^{1}\right\| \leqslant \frac{2}{\nu}\left\|R^{0}\right\| .
$$

Combining (2.7), (3.15), (3.37), (3.39) and (3.42), and recalling Lemma 2.1, we have

$$
\begin{aligned}
\left\|e^{1}\right\|_{l^{\infty}}^{2} & \leqslant\left(C_{\alpha, \beta}\right)^{2}\left(\left\|e^{1}\right\|^{2}+\left|e^{1}\right|_{H_{h}^{\alpha, \beta}}^{2}+\left|e^{1}\right|_{H_{h}^{\frac{\alpha}{2}}, \frac{\beta}{2}}^{2}\right) \\
& \leqslant \frac{\left(C_{\alpha, \beta}\right)^{2}}{\left(\widetilde{C}_{\alpha, \beta}\right)^{2}}\left(\left\|e^{1}\right\|^{2}+\left\|\Lambda^{\alpha, \beta} e^{1}\right\|^{2}+\left\|\left(\delta_{x}^{\alpha}+\delta_{y}^{\beta}\right) e^{1}\right\|^{2}\right) \\
& :=c_{12}^{2}\left(\tau^{2}+h_{x}^{2}+h_{y}^{2}\right)^{2} .
\end{aligned}
$$

Therefore, (3.28) holds for the case $n=1$. Next, we assume that 3.28 is true for $1 \leqslant n \leqslant$ $l,(1 \leqslant l \leqslant N-1)$. Then we seek to prove that (3.28) holds for $n=l+1$. By the induction, it yields

$$
\left\|e^{n}\right\|_{l^{\infty}} \leqslant c_{12}\left(\tau^{2}+h_{x}^{2}+h_{y}^{2}\right), \quad 1 \leqslant n \leqslant l
$$


and

$$
\left\|u^{n}\right\|_{l^{\infty}} \leqslant\left\|e^{n}\right\|_{l^{\infty}}+\left\|U^{n}\right\|_{l^{\infty}} \leqslant C_{u}+c_{12}\left(\tau_{0}^{2}+2 h_{0}^{2}\right) \leqslant C_{u}+1, \quad 1 \leqslant n \leqslant l,
$$

for $\tau \leqslant \tau_{0}, h_{x} \leqslant h_{0}, h_{y} \leqslant h_{0}$, where $\tau_{0}^{2}+2 h_{0}^{2} \leqslant \frac{1}{c_{12}}$.

Step 1: Proof in $\|\cdot\|$ norm. We take the discrete inner product of (3.30) with $e^{\bar{n}}$ to have

$$
\left(\Delta_{t} e^{n}, e^{\bar{n}}\right)+(\nu+\mathbf{i} \eta)\left\|\Lambda^{\alpha, \beta} e^{\bar{n}}\right\|^{2}+(\kappa+\mathbf{i} \zeta)\left(p^{n}, e^{\bar{n}}\right)-\gamma\left\|e^{\bar{n}}\right\|^{2}=\left(R^{n}, e^{\bar{n}}\right) .
$$

Noticing that

$$
\begin{aligned}
& \operatorname{Re}\left(\Delta_{t} e^{n}, e^{\bar{n}}\right)=\frac{1}{4 \tau}\left(\left\|e^{n+1}\right\|^{2}-\left\|e^{n-1}\right\|^{2}\right), \\
& \left(p^{n}, e^{\bar{n}}\right)=\left(\left|u^{n}\right|^{2} e^{\bar{n}}, e^{\bar{n}}\right)+\left(U^{n} U^{\bar{n}}\left(e^{n}\right)^{*}, e^{\bar{n}}\right)+\left(U^{\bar{n}}\left(u^{n}\right)^{*} e^{n}, e^{\bar{n}}\right),
\end{aligned}
$$

and taking the real part of (3.45), substituting (3.46)-(3.47) into (3.45), we have

$$
\begin{aligned}
& \frac{1}{4 \tau}\left(\left\|e^{n+1}\right\|^{2}-\left\|e^{n-1}\right\|^{2}\right)+\nu\left\|\Lambda^{\alpha, \beta} e^{\bar{n}}\right\|^{2}+\kappa\left(\left|u^{n}\right|^{2} e^{\bar{n}}, e^{\bar{n}}\right) \\
= & -\operatorname{Re}\left\{(\kappa+\mathbf{i} \zeta)\left[\left(U^{n} U^{\bar{n}}\left(e^{n}\right)^{*}, e^{\bar{n}}\right)+\left(U^{\bar{n}}\left(u^{n}\right)^{*} e^{n}, e^{\bar{n}}\right)\right]\right\}+\gamma\left\|e^{\bar{n}}\right\|^{2}+\operatorname{Re}\left(R^{n}, e^{\bar{n}}\right) .
\end{aligned}
$$

From (3.44), we have

$$
\begin{aligned}
& \frac{1}{4 \tau}\left(\left\|e^{n+1}\right\|^{2}-\left\|e^{n-1}\right\|^{2}\right) \\
\leqslant & \sqrt{\kappa^{2}+\zeta^{2}}\left(\left|\left(U^{n} U^{\bar{n}}\left(e^{n}\right)^{*}, e^{\bar{n}}\right)\right|+\left|\left(U^{\bar{n}}\left(u^{n}\right)^{*} e^{n}, e^{\bar{n}}\right)\right|\right)+|\gamma| \cdot\left\|e^{\bar{n}}\right\|^{2}+\left\|R^{n}\right\| \cdot\left\|e^{\bar{n}}\right\| \\
\leqslant & \frac{1}{2} \sqrt{\kappa^{2}+\zeta^{2}} C_{u}\left(2 C_{u}+1\right)\left(\left\|e^{n}\right\|^{2}+\left\|e^{\bar{n}}\right\|^{2}\right)+|\gamma| \cdot\left\|e^{\bar{n}}\right\|^{2}+\frac{1}{2}\left\|e^{\bar{n}}\right\|^{2}+\frac{1}{2}\left\|R^{n}\right\|^{2} \\
\leqslant & c_{1}\left(\left\|e^{n+1}\right\|^{2}+\left\|e^{n}\right\|^{2}+\left\|e^{n-1}\right\|^{2}\right)+\frac{1}{2}\left\|R^{n}\right\|^{2}, \quad 1 \leqslant n \leqslant l .
\end{aligned}
$$

Therefore, we have

$$
\left\|e^{n+1}\right\|^{2}-\left\|e^{n-1}\right\|^{2} \leqslant 4 c_{1} \tau\left(\left\|e^{n+1}\right\|^{2}+\left\|e^{n}\right\|^{2}+\left\|e^{n-1}\right\|^{2}\right)+2 \tau\left\|R^{n}\right\|^{2}, \quad 1 \leqslant n \leqslant l .
$$

Let

$$
E_{n}:=\left\|e^{n+1}\right\|^{2}+\left\|e^{n}\right\|^{2},
$$

and (3.50) becomes

$$
E_{n}-E_{n-1} \leqslant 4 c_{1} \tau\left(E_{n}+E_{n-1}\right)+2 \tau\left\|R^{n}\right\|^{2}, \quad 1 \leqslant n \leqslant l,
$$

and

$$
\left(1-4 c_{1} \tau\right) E_{n} \leqslant\left(1+4 c_{1} \tau\right) E_{n-1}+2 \tau\left\|R^{n}\right\|^{2}, \quad 1 \leqslant n \leqslant l .
$$

When $\tau<\tau_{0}=\frac{1}{8 c_{1}}$, we have $1-4 c_{1} \tau \geqslant \frac{1}{2}$. Thus,

$$
E_{n} \leqslant\left(1+16 c_{1} \tau\right) E_{n-1}+4 \tau\left\|R^{n}\right\|^{2}, \quad 1 \leqslant n \leqslant l .
$$

By (3.37), we have

$$
E_{0}=\left\|e^{1}\right\|^{2} \leqslant 4 \tau^{2}\left\|R^{0}\right\|^{2} .
$$


Using Gronwall's inequality in Lemma 3.6 for (3.53) and recalling (3.11), (3.15), we have

$$
\begin{aligned}
\left\|e^{n+1}\right\|^{2}+\left\|e^{n}\right\|^{2}=E_{n} & \leqslant \exp \left(16 c_{1} n \tau\right)\left(E_{0}+\frac{\left\|R^{n}\right\|^{2}}{4 c_{1}}\right) \\
& \leqslant c_{2} \exp \left(16 c_{1} T\right)\left(\tau^{2}+h_{x}^{2}+h_{y}^{2}\right)^{2} \\
& =c_{3}\left(\tau^{2}+h_{x}^{2}+h_{y}^{2}\right)^{2}, \quad 1 \leqslant n \leqslant l .
\end{aligned}
$$

Step 2: Taking the discrete inner product of (3.30) with $-\left(\delta_{x}^{\alpha}+\delta_{y}^{\beta}\right) e^{\bar{n}}$, we have

$$
\begin{aligned}
& -\left(\Delta_{t} e^{n},\left(\delta_{x}^{\alpha}+\delta_{y}^{\beta}\right) e^{\bar{n}}\right)+(\nu+\mathbf{i} \eta)\left\|\left(\delta_{x}^{\alpha}+\delta_{y}^{\beta}\right) e^{\bar{n}}\right\|^{2} \\
& -(\kappa+\mathbf{i} \zeta)\left(p^{n},\left(\delta_{x}^{\alpha}+\delta_{y}^{\beta}\right) e^{\bar{n}}\right)+\gamma\left(e^{\bar{n}},\left(\delta_{x}^{\alpha}+\delta_{y}^{\beta}\right) e^{\bar{n}}\right)=-\left(R^{n},\left(\delta_{x}^{\alpha}+\delta_{y}^{\beta}\right) e^{\bar{n}}\right) .
\end{aligned}
$$

Noticing that

$$
\begin{aligned}
& -\operatorname{Re}\left(\Delta_{t} e^{n},\left(\delta_{x}^{\alpha}+\delta_{y}^{\beta}\right) e^{\bar{n}}\right)=\frac{1}{4 \tau}\left(\left\|\Lambda^{\alpha, \beta} e^{n+1}\right\|^{2}-\left\|\Lambda^{\alpha, \beta} e^{n-1}\right\|^{2}\right), \\
& -\left(p^{n},\left(\delta_{x}^{\alpha}+\delta_{y}^{\beta}\right) e^{\bar{n}}\right)=\left(\left|u^{n}\right|^{2} \Lambda^{\alpha, \beta} e^{\bar{n}}, \Lambda^{\alpha, \beta} e^{\bar{n}}\right)-\left(U^{n} U^{\bar{n}}\left(e^{n}\right)^{*}+U^{\bar{n}}\left(u^{n}\right)^{*} e^{n},\left(\delta_{x}^{\alpha}+\delta_{y}^{\beta}\right) e^{\bar{n}}\right) .
\end{aligned}
$$

Taking the real part of (3.56), and substituting (3.57)-(3.58) into (3.56), we recover

$$
\begin{aligned}
& \frac{1}{4 \tau}\left(\left\|\Lambda^{\alpha, \beta} e^{n+1}\right\|^{2}-\left\|\Lambda^{\alpha, \beta} e^{n-1}\right\|^{2}\right)+\nu\left\|\left(\delta_{x}^{\alpha}+\delta_{y}^{\beta}\right) e^{\bar{n}}\right\|^{2} \\
= & \operatorname{Re}\left\{( \kappa + \mathbf { i } \zeta ) \left[\left(U^{n} U^{\bar{n}}\left(e^{n}\right)^{*},\left(\delta_{x}^{\alpha}+\delta_{y}^{\beta}\right) e^{\bar{n}}\right)+\left(U^{\bar{n}}\left(u^{n}\right)^{*} e^{n},\left(\delta_{x}^{\alpha}+\delta_{y}^{\beta}\right) e^{\bar{n}}\right)\right.\right. \\
& \left.\left.+\left(\left|u^{n}\right|^{2} e^{\bar{n}},\left(\delta_{x}^{\alpha}+\delta_{y}^{\beta}\right) e^{\bar{n}}\right)\right]\right\}-\gamma\left(e^{\bar{n}},\left(\delta_{x}^{\alpha}+\delta_{y}^{\beta}\right) e^{\bar{n}}\right)-\left(R^{n},\left(\delta_{x}^{\alpha}+\delta_{y}^{\beta}\right) e^{\bar{n}}\right) \\
\leqslant & \sqrt{\kappa^{2}+\zeta^{2}}\left[C_{u}\left(2 C_{u}+1\right)\left\|e^{n}\right\| \cdot\left\|\left(\delta_{x}^{\alpha}+\delta_{y}^{\beta}\right) e^{\bar{n}}\right\|+\left(C_{u}+1\right)^{2}\left\|e^{\bar{n}}\right\| \cdot\left\|\left(\delta_{x}^{\alpha}+\delta_{y}^{\beta}\right) e^{\bar{n}}\right\|\right] \\
& +|\gamma| \cdot\left\|e^{\bar{n}}\right\| \cdot\left\|\left(\delta_{x}^{\alpha}+\delta_{y}^{\beta}\right) e^{\bar{n}}\right\|+\left\|R^{n}\right\| \cdot\left\|\left(\delta_{x}^{\alpha}+\delta_{y}^{\beta}\right) e^{\bar{n}}\right\| \\
\leqslant & \frac{1}{2}\left\{\frac{\sqrt{\kappa^{2}+\zeta^{2}}}{2}\left[C_{u}\left(2 C_{u}+1\right)+\left(C_{u}+1\right)^{2}\right]+\frac{|\gamma|}{2}+\frac{1}{2}\right\} \\
& \times\left(\left\|\left(\delta_{x}^{\alpha}+\delta_{y}^{\beta}\right) e^{n+1}\right\|^{2}+\left\|\left(\delta_{x}^{\alpha}+\delta_{y}^{\beta}\right) e^{n-1}\right\|^{2}\right) \\
& +\frac{|\gamma|}{4}\left(\left\|e^{n+1}\right\|^{2}+\left\|e^{n-1}\right\|^{2}\right)+\frac{\sqrt{\kappa^{2}+\zeta^{2}}}{2} C_{u}\left(2 C_{u}+1\right)\left\|e^{n}\right\|^{2}+\frac{1}{2}\left\|R^{n}\right\|^{2} \\
\leqslant & c_{1}\left(\left\|e^{n+1}\right\|^{2}+\left\|e^{n}\right\|^{2}+\left\|e^{n-1}\right\|^{2}\right) \\
& +c_{1}\left(\left\|\left(\delta_{x}^{\alpha}+\delta_{y}^{\beta}\right) e^{n+1}\right\|^{2}+\left\|\left(\delta_{x}^{\alpha}+\delta_{y}^{\beta}\right) e^{n-1}\right\|^{2}\right)+\frac{1}{2}\left\|R^{n}\right\|^{2}, \quad 1 \leqslant n \leqslant l .
\end{aligned}
$$

Following the result in Step 1, we have

$$
\left\|e^{n+1}\right\|^{2}+\left\|e^{n}\right\|^{2}+\left\|e^{n-1}\right\|^{2} \leqslant E_{n}+E_{n-1} \leqslant 2 c_{3}\left(\tau^{2}+h_{x}^{2}+h_{y}^{2}\right)^{2}, \quad 1 \leqslant n \leqslant l .
$$

Again, we compute the discrete inner product of (3.30) with $-\left(\delta_{x}^{\alpha}+\delta_{y}^{\beta}\right) \Delta_{t} e^{n}$ to recover

$$
\begin{aligned}
& \left\|\Lambda^{\alpha, \beta} \Delta_{t} e^{n}\right\|^{2}+\frac{\nu+\mathbf{i} \eta}{4 \tau}\left(\left\|\left(\delta_{x}^{\alpha}+\delta_{y}^{\beta}\right) e^{n+1}\right\|^{2}-\left\|\left(\delta_{x}^{\alpha}+\delta_{y}^{\beta}\right) e^{n-1}\right\|^{2}\right) \\
& -(\kappa+\mathbf{i} \zeta)\left(p^{n},\left(\delta_{x}^{\alpha}+\delta_{y}^{\beta}\right) \Delta_{t} e^{n}\right)+\gamma\left(e^{\bar{n}},\left(\delta_{x}^{\alpha}+\delta_{y}^{\beta}\right) \Delta_{t} e^{n}\right)=-\left(R^{n},\left(\delta_{x}^{\alpha}+\delta_{y}^{\beta}\right) \Delta_{t} e^{n}\right) .
\end{aligned}
$$


Taking the real part of (3.61), and using the Cauchy-Schwarz inequality, we have

$$
\begin{aligned}
& \left\|\Lambda^{\alpha, \beta} \Delta_{t} e^{n}\right\|^{2}+\frac{\nu}{4 \tau}\left(\left\|\left(\delta_{x}^{\alpha}+\delta_{y}^{\beta}\right) e^{n+1}\right\|^{2}-\left\|\left(\delta_{x}^{\alpha}+\delta_{y}^{\beta}\right) e^{n-1}\right\|^{2}\right) \\
\leqslant & \operatorname{Re}\left\{(\kappa+\mathbf{i} \zeta)\left|\left(p^{n},\left(\delta_{x}^{\alpha}+\delta_{y}^{\beta}\right) \Delta_{t} e^{n}\right)\right|\right\} \\
& +\operatorname{Re}\left|\left(R^{n},\left(\delta_{x}^{\alpha}+\delta_{y}^{\beta}\right) \Delta_{t} e^{n}\right)\right|+|\gamma| \varepsilon \cdot\left\|\Lambda^{\alpha, \beta} e^{\bar{n}}\right\|^{2}+\frac{|\gamma|}{4 \varepsilon} \cdot\left\|\Lambda^{\alpha, \beta} \Delta_{t} e^{n}\right\|^{2} .
\end{aligned}
$$

Taking $\varepsilon=\frac{|\gamma|}{4}$, we recover

$$
\begin{aligned}
& \frac{\nu}{4 \tau}\left(\left\|\left(\delta_{x}^{\alpha}+\delta_{y}^{\beta}\right) e^{n+1}\right\|^{2}-\left\|\left(\delta_{x}^{\alpha}+\delta_{y}^{\beta}\right) e^{n-1}\right\|^{2}\right) \\
\leqslant & \operatorname{Re}\left\{(\kappa+\mathbf{i} \zeta)\left|\left(p^{n},\left(\delta_{x}^{\alpha}+\delta_{y}^{\beta}\right) \Delta_{t} e^{n}\right)\right|\right\}+\operatorname{Re}\left|\left(R^{n},\left(\delta_{x}^{\alpha}+\delta_{y}^{\beta}\right) \Delta_{t} e^{n}\right)\right| \\
& +\frac{|\gamma|^{2}}{8}\left(\left\|\Lambda^{\alpha, \beta} e^{n+1}\right\|^{2}+\left\|\Lambda^{\alpha, \beta} e^{n-1}\right\|^{2}\right) .
\end{aligned}
$$

Combining (3.59) and (3.63), we have

$$
\begin{aligned}
& \left(\left\|\left(\delta_{x}^{\alpha}+\delta_{y}^{\beta}\right) e^{n+1}\right\|^{2}+\left\|\Lambda^{\alpha, \beta} e^{n+1}\right\|^{2}\right)-\left(\left\|\left(\delta_{x}^{\alpha}+\delta_{y}^{\beta}\right) e^{n-1}\right\|^{2}+\left\|\Lambda^{\alpha, \beta} e^{n-1}\right\|^{2}\right) \\
\leqslant & 2 \tau\left\|R^{n}\right\|^{2}+4 c_{1} \tau\left(\left\|e^{n+1}\right\|^{2}+\left\|e^{n}\right\|^{2}+\left\|e^{n-1}\right\|^{2}+\left\|\left(\delta_{x}^{\alpha}+\delta_{y}^{\beta}\right) e^{n+1}\right\|^{2}+\left\|\left(\delta_{x}^{\alpha}+\delta_{y}^{\beta}\right) e^{n-1}\right\|^{2}\right) \\
& +\frac{4 \tau}{\nu} \operatorname{Re}\left\{(\kappa+\mathbf{i} \zeta)\left|\left(p^{n},\left(\delta_{x}^{\alpha}+\delta_{y}^{\beta}\right) \Delta_{t} e^{n}\right)\right|\right\}+\frac{4 \tau}{\nu} \operatorname{Re}\left|\left(R^{n},\left(\delta_{x}^{\alpha}+\delta_{y}^{\beta}\right) \Delta_{t} e^{n}\right)\right| \\
& +\frac{|\gamma|^{2} \tau}{2 \nu}\left(\left\|\Lambda^{\alpha, \beta} e^{n+1}\right\|^{2}+\left\|\Lambda^{\alpha, \beta} e^{n-1}\right\|^{2}\right) .
\end{aligned}
$$

Denote

$$
F_{n}=\left\|\left(\delta_{x}^{\alpha}+\delta_{y}^{\beta}\right) e^{n+1}\right\|^{2}+\left\|\left(\delta_{x}^{\alpha}+\delta_{y}^{\beta}\right) e^{n}\right\|^{2}+\left\|\Lambda^{\alpha, \beta} e^{n+1}\right\|^{2}+\left\|\Lambda^{\alpha, \beta} e^{n}\right\|^{2} .
$$

Then (3.64) becomes

$$
\begin{aligned}
& F_{n}-F_{n-1} \\
\leqslant & 4 c_{4} \tau\left(F_{n}+F_{n-1}\right)+\frac{4 \tau}{\nu} \operatorname{Re}\left\{(\kappa+\mathbf{i} \zeta)\left|\left(p^{n},\left(\delta_{x}^{\alpha}+\delta_{y}^{\beta}\right) \Delta_{t} e^{n}\right)\right|\right\} \\
& +\frac{4 \tau}{\nu} \operatorname{Re}\left|\left(R^{n},\left(\delta_{x}^{\alpha}+\delta_{y}^{\beta}\right) \Delta_{t} e^{n}\right)\right|+4 c_{1} \tau\left(\left\|e^{n+1}\right\|^{2}+\left\|e^{n}\right\|^{2}+\left\|e^{n-1}\right\|^{2}\right)+2 \tau\left\|R^{n}\right\|^{2} .
\end{aligned}
$$

When $\tau \leqslant \tau_{0}=\frac{1}{8 c_{4}}$, we have

$$
\begin{aligned}
F_{n} \leqslant & \left(1+16 c_{4} \tau\right) F_{n-1}+\frac{8 \tau}{\nu} \operatorname{Re}\left\{(\kappa+\mathbf{i} \zeta)\left|\left(p^{n},\left(\delta_{x}^{\alpha}+\delta_{y}^{\beta}\right) \Delta_{t} e^{n}\right)\right|\right\}+\frac{8 \tau}{\nu} \operatorname{Re}\left|\left(R^{n},\left(\delta_{x}^{\alpha}+\delta_{y}^{\beta}\right) \Delta_{t} e^{n}\right)\right| \\
& +8 c_{1} \tau\left(\left\|e^{n+1}\right\|^{2}+\left\|e^{n}\right\|^{2}+\left\|e^{n-1}\right\|^{2}\right)+4 \tau\left\|R^{n}\right\|^{2}, \quad 1 \leqslant n \leqslant l .
\end{aligned}
$$

Replacing $n$ with $k$ in (3.66) and summing over $k$, we recover

$$
\begin{aligned}
F_{n} \leqslant & 16 c_{4} \tau \sum_{k=1}^{n} F_{k-1}+F_{0}+\frac{8 \tau}{\nu} \sum_{k=1}^{n} \operatorname{Re}\left\{(\kappa+\mathbf{i} \zeta)\left|\left(p^{k},\left(\delta_{x}^{\alpha}+\delta_{y}^{\beta}\right) \Delta_{t} e^{k}\right)\right|\right\} \\
& +\frac{8 \tau}{\nu} \sum_{k=1}^{n} \operatorname{Re}\left|\left(R^{k},\left(\delta_{x}^{\alpha}+\delta_{y}^{\beta}\right) \Delta_{t} e^{k}\right)\right|+8 c_{1} \tau \sum_{k=1}^{n}\left(\left\|e^{k+1}\right\|^{2}+\left\|e^{k}\right\|^{2}+\left\|e^{k-1}\right\|^{2}\right)
\end{aligned}
$$




$$
+4 \tau \sum_{k=1}^{n}\left\|R^{k}\right\|^{2}
$$

Step 3: We estimate each term in (3.67). Noticing 3.11) and (3.60, we have

$$
\begin{aligned}
& 8 c_{1} \tau \sum_{k=1}^{n}\left(\left\|e^{k+1}\right\|^{2}+\left\|e^{k}\right\|^{2}+\left\|e^{k-1}\right\|^{2}\right)+4 \tau \sum_{k=1}^{n}\left\|R^{k}\right\|^{2} \\
\leqslant & \left(16 c_{1} c_{3}+4 C_{R}^{2}|\Omega|\right) T\left(\tau^{2}+h_{x}^{2}+h_{y}^{2}\right)^{2}:=c_{5}\left(\tau^{2}+h_{x}^{2}+h_{y}^{2}\right)^{2} .
\end{aligned}
$$

In addition, for the fourth term on the right hand side of (3.67), we have

$$
\begin{aligned}
& \frac{8 \tau}{\nu} \sum_{k=1}^{n} \operatorname{Re}\left(R^{k},\left(\delta_{x}^{\alpha}+\delta_{y}^{\beta}\right) \Delta_{t} e^{k}\right) \\
= & \frac{8 \tau}{\nu} \sum_{k=2}^{n+1} \frac{1}{2 \tau} \operatorname{Re}\left(R^{k+1},\left(\delta_{x}^{\alpha}+\delta_{y}^{\beta}\right) e^{k}\right)-\frac{8 \tau}{\nu} \sum_{k=0}^{n-1} \frac{1}{2 \tau} \operatorname{Re}\left(R^{k+1},\left(\delta_{x}^{\alpha}+\delta_{y}^{\beta}\right) e^{k}\right) \\
= & \frac{4}{\nu} \operatorname{Re}\left\{\left(R^{n},\left(\delta_{x}^{\alpha}+\delta_{y}^{\beta}\right) e^{n+1}\right)+\left(R^{n-1},\left(\delta_{x}^{\alpha}+\delta_{y}^{\beta}\right) e^{n}\right)-\left(R^{2},\left(\delta_{x}^{\alpha}+\delta_{y}^{\beta}\right) e^{1}\right)\right\} \\
& -\frac{8 \tau}{\nu} \sum_{k=2}^{n-1} \operatorname{Re}\left(\Delta_{t} R^{k},\left(\delta_{x}^{\alpha}+\delta_{y}^{\beta}\right) e^{k}\right) .
\end{aligned}
$$

Using the Cauchy-Schwarz inequality, and recalling (3.11), (3.12) and (3.42), we have

$$
\begin{aligned}
& \frac{8 \tau}{\nu} \sum_{k=1}^{n} \operatorname{Re}\left|\left(R^{k},\left(\delta_{x}^{\alpha}+\delta_{y}^{\beta}\right) \Delta_{t} e^{k}\right)\right| \\
\leqslant & {\left[\frac{1}{\nu}\left\|R^{n}\right\| \cdot\left\|\left(\delta_{x}^{\alpha}+\delta_{y}^{\beta}\right) e^{n+1}\right\|+\frac{1}{\nu}\left\|R^{n-1}\right\| \cdot\left\|\left(\delta_{x}^{\alpha}+\delta_{y}^{\beta}\right) e^{n}\right\|+\frac{1}{\nu}\left\|R^{2}\right\| \cdot\left\|\left(\delta_{x}^{\alpha}+\delta_{y}^{\beta}\right) e^{1}\right\|\right] } \\
& \left.+\frac{8 \tau}{\nu} \sum_{k=2}^{n-1}\left\|\Delta_{t} R^{k}\right\| \cdot \|\left(\delta_{x}^{\alpha}+\delta_{y}^{\beta}\right) e^{k}\right) \| \\
\leqslant & \frac{16}{\nu^{2}}\left\|R^{n}\right\|^{2}+\frac{16}{\nu^{2}}\left\|R^{n-1}\right\|^{2}+\frac{2}{\nu^{2}}\left\|R^{2}\right\|^{2}+2\left\|\left(\delta_{x}^{\alpha}+\delta_{y}^{\beta}\right) e^{1}\right\|^{2}+\frac{1}{4}\left\|\left(\delta_{x}^{\alpha}+\delta_{y}^{\beta}\right) e^{n+1}\right\|^{2} \\
& \left.+\frac{1}{4}\left\|\left(\delta_{x}^{\alpha}+\delta_{y}^{\beta}\right) e^{n}\right\|^{2}+4 \tau \sum_{k=2}^{n-1} \frac{1}{\nu^{2}}\left\|\Delta_{t} R^{k}\right\|^{2}+4 \tau \sum_{k=2}^{n-1} \|\left(\delta_{x}^{\alpha}+\delta_{y}^{\beta}\right) e^{k}\right) \|^{2} \\
\leqslant & \left.4 \tau \sum_{k=2}^{n-1} \|\left(\delta_{x}^{\alpha}+\delta_{y}^{\beta}\right) e^{k}\right)\left\|^{2}+\frac{1}{4}\right\|\left(\delta_{x}^{\alpha}+\delta_{y}^{\beta}\right) e^{n+1}\left\|^{2}+\frac{1}{4}\right\|\left(\delta_{x}^{\alpha}+\delta_{y}^{\beta}\right) e^{n} \|^{2}+c_{6}\left(\tau^{2}+h_{x}^{2}+h_{y}^{2}\right)^{2} .
\end{aligned}
$$

Next, the third term on the right hand side of (3.67) can be estimated as follows

$$
\begin{aligned}
& \frac{8 \tau}{\nu} \sum_{k=1}^{n} \operatorname{Re}\left\{(\kappa+\mathbf{i} \zeta)\left(p^{k},\left(\delta_{x}^{\alpha}+\delta_{y}^{\beta}\right) \Delta_{t} e^{k}\right)\right\} \\
= & \frac{4}{\nu} \operatorname{Re}\left\{(\kappa+\mathbf{i} \zeta)\left[\left(p^{n},\left(\delta_{x}^{\alpha}+\delta_{y}^{\beta}\right) e^{n+1}\right)+\left(p^{n-1},\left(\delta_{x}^{\alpha}+\delta_{y}^{\beta}\right) e^{n}\right)-\left(p^{2},\left(\delta_{x}^{\alpha}+\delta_{y}^{\beta}\right) e^{1}\right)\right]\right\}
\end{aligned}
$$




$$
-\frac{8 \tau}{\nu} \sum_{k=2}^{n-1} \operatorname{Re}\left\{(\kappa+\mathbf{i} \zeta)\left(\Delta_{t} p^{k},\left(\delta_{x}^{\alpha}+\delta_{y}^{\beta}\right) e^{k}\right)\right\}
$$

where

$$
\begin{aligned}
p^{k}= & \left|U^{k}\right|^{2} U^{\bar{k}}-\left|u^{k}\right|^{2} u^{\bar{k}} \\
= & \left(U^{k}\right)^{*} U^{\bar{k}} e^{k}+U^{k} U^{\bar{k}}\left(e^{k}\right)^{*}+U^{k}\left(U^{k}\right)^{*} e^{\bar{k}}-\left(e^{k}\right)^{*} e^{\bar{k}} U^{k}-e^{k} e^{\bar{k}}\left(U^{k}\right)^{*} \\
& -e^{k}\left(e^{k}\right)^{*} U^{\bar{k}}+e^{k}\left(e^{k}\right)^{*} e^{\bar{k}}
\end{aligned}
$$

and

$$
\begin{aligned}
\Delta_{t} p^{k}= & \Delta_{t}\left(\left(U^{k}\right)^{*} U^{\bar{k}} e^{k}\right)+\Delta_{t}\left(U^{k} U^{\bar{k}}\left(e^{k}\right)^{*}\right)+\Delta_{t}\left(U^{k}\left(U^{k}\right)^{*} e^{\bar{k}}\right)-\Delta_{t}\left(\left(e^{k}\right)^{*} e^{\bar{k}} U^{k}\right) \\
& -\Delta_{t}\left(e^{k} e^{\bar{k}}\left(U^{k}\right)^{*}\right)-\Delta_{t}\left(e^{k}\left(e^{k}\right)^{*} U^{\bar{k}}\right)+\Delta_{t}\left(e^{k}\left(e^{k}\right)^{*} e^{\bar{k}}\right)=: \sum_{i=1}^{7} J_{i} .
\end{aligned}
$$

Clearly, we have

$$
\left\|\Delta_{t} p^{k}\right\| \leqslant \sum_{i=1}^{7}\left\|J_{i}\right\|
$$

Following (3.30), we have

$$
\begin{aligned}
\left\|\Delta_{t} e^{k}\right\| \leqslant & \left\|(\nu+\mathbf{i} \eta)\left(\delta_{x}^{\alpha}+\delta_{y}^{\beta}\right) e^{\bar{n}}\right\|+\left\|(\kappa+\mathbf{i} \zeta) p^{k}\right\|+|\gamma| \cdot\left\|e^{\bar{n}}\right\|+\left\|R^{k}\right\| \\
\leqslant & \frac{\sqrt{\nu^{2}+\eta^{2}}}{2}\left(\left\|\left(\delta_{x}^{\alpha}+\delta_{y}^{\beta}\right) e^{n+1}\right\|+\left\|\left(\delta_{x}^{\alpha}+\delta_{y}^{\beta}\right) e^{n-1}\right\|\right)+\sqrt{\kappa^{2}+\zeta^{2}}\left\|p^{k}\right\| \\
& +\frac{|\gamma|}{2}\left(\left\|e^{n+1}\right\|+\left\|e^{n-1}\right\|\right)+\left\|R^{k}\right\| .
\end{aligned}
$$

Using the result of Step 1, we have $\left\|e^{n+1}\right\|^{2}+\left\|e^{n}\right\|^{2} \leqslant c_{3}\left(\tau^{2}+h_{x}^{2}+h_{y}^{2}\right)^{2} \leqslant 1$ when $\tau$, $h_{x}, h_{y}<\frac{1}{\sqrt[4]{9 c_{3}}}$. With the help of Lemma 3.4 and (3.73), the seven terms in (3.72) can be bounded, respectively, as follows

$$
\begin{aligned}
\left\|J_{1}\right\| & =\left\|\Delta_{t}\left(U^{k}\right)^{*} e^{\bar{k}} U^{\hat{k}}+\Delta_{t} e^{k}\left(U^{\bar{k}}\right)^{*} U^{\hat{k}}+\Delta_{t} U^{\bar{k}}\left(U^{\bar{k}}\right)^{*} e^{\bar{k}}\right\| \\
& \leqslant\left\|u_{t}\right\|_{\infty} \cdot\|u\|_{\infty} \cdot\left\|e^{\bar{k}}\right\|+\|u\|_{\infty}^{2} \cdot\left\|\Delta_{t} e^{k}\right\|+\left\|u_{t}\right\|_{\infty} \cdot\|u\|_{\infty} \cdot\left\|e^{\bar{k}}\right\| \\
& \leqslant c_{7}\left(\left\|e^{k+1}\right\|+\left\|e^{k-1}\right\|+\left\|R^{k}\right\|+\left\|p^{k}\right\|+\left\|\left(\delta_{x}^{\alpha}+\delta_{y}^{\beta}\right) e^{k+1}\right\|+\left\|\left(\delta_{x}^{\alpha}+\delta_{y}^{\beta}\right) e^{k-1}\right\|\right) \\
\left\|J_{2}\right\| & =\left\|\Delta_{t} U^{k} U^{\hat{n}}\left(e^{\bar{k}}\right)^{*}+\Delta_{t}\left(e^{k}\right)^{*} U^{\bar{k}} U^{\hat{k}}+\Delta_{t} U^{\bar{k}} U^{\bar{k}}\left(e^{\bar{k}}\right)^{*}\right\| \\
& \leqslant\left\|u_{t}\right\|_{\infty} \cdot\|u\|_{\infty} \cdot\left\|e^{\bar{k}}\right\|+\|u\|_{\infty}^{2} \cdot\left\|\Delta_{t} e^{k}\right\|+\left\|u_{t}\right\|_{\infty} \cdot\|u\|_{\infty} \cdot\left\|e^{\bar{k}}\right\| \\
& \leqslant c_{7}\left(\left\|e^{k+1}\right\|+\left\|e^{k-1}\right\|+\left\|R^{k}\right\|+\left\|p^{k}\right\|+\left\|\left(\delta_{x}^{\alpha}+\delta_{y}^{\beta}\right) e^{k+1}\right\|+\left\|\left(\delta_{x}^{\alpha}+\delta_{y}^{\beta}\right) e^{k-1}\right\|\right), \\
\left\|J_{3}\right\| & =\left\|\Delta_{t} U^{k}\left(U^{k}\right)^{*} e^{\hat{k}}+\Delta_{t}\left(U^{k}\right)^{*} U^{\bar{k}} e^{\hat{k}}+U^{\bar{k}}\left(U^{\bar{k}}\right)^{*} \Delta_{t} e^{\bar{k}}\right\| \\
& \leqslant\left\|u_{t}\right\|_{\infty} \cdot\|u\|_{\infty} \cdot\left\|e^{\hat{k}}\right\|+\left\|u_{t}\right\|_{\infty} \cdot\|u\|_{\infty} \cdot\left\|e^{\hat{k}}\right\|+\|u\|_{\infty}^{2} \cdot\left\|\Delta_{t} e^{\bar{k}}\right\| \\
& \leqslant c_{7}\left(\left\|e^{k+2}\right\|+\left\|e^{k}\right\|+\left\|e^{k-2}\right\|+\left\|R^{k+1}\right\|+\left\|R^{k-1}\right\|\right.
\end{aligned}
$$


$\left.+\left\|p^{k+1}\right\|+\left\|p^{k-1}\right\|+\left\|\left(\delta_{x}^{\alpha}+\delta_{y}^{\beta}\right) e^{k+2}\right\|+\left\|\left(\delta_{x}^{\alpha}+\delta_{y}^{\beta}\right) e^{k}\right\|+\left\|\left(\delta_{x}^{\alpha}+\delta_{y}^{\beta}\right) e^{k-2}\right\|\right)$,

$$
\left\|J_{4}\right\|=\left\|\Delta_{t} U^{k}\left(e^{\bar{k}}\right)^{*} e^{\hat{k}}+\Delta_{t}\left(e^{k}\right)^{*} U^{\bar{k}} e^{\hat{k}}+U^{\bar{k}}\left(e^{\bar{k}}\right)^{*} \Delta_{t} e^{\bar{k}}\right\|
$$$$
\leqslant\left\|u_{t}\right\|_{\infty} \cdot\left\|e^{\bar{k}}\right\| \cdot\left\|e^{\hat{k}}\right\|+\|u\|_{\infty} \cdot\left\|e^{\hat{k}}\right\| \cdot\left\|\Delta_{t} e^{k}\right\|+\|u\|_{\infty} \cdot\left\|e^{\bar{k}}\right\| \cdot\left\|\Delta_{t} e^{\bar{k}}\right\|
$$$$
\leqslant c_{7}\left(\left\|e^{\hat{k}}\right\|+\left\|\Delta_{t} e^{k}\right\|+\left\|\Delta_{t} e^{\bar{k}}\right\|\right)
$$$$
\leqslant c_{7}\left(\left\|e^{k+2}\right\|+\left\|e^{k+1}\right\|+\left\|e^{k}\right\|+\left\|e^{k-1}\right\|+\left\|e^{k-2}\right\|\right.
$$$$
+\left\|R^{k+1}\right\|+\left\|R^{k}\right\|+\left\|R^{k-1}\right\|+\left\|p^{k+1}\right\|+\left\|p^{k}\right\|+\left\|p^{k-1}\right\|
$$$$
+\left\|\left(\delta_{x}^{\alpha}+\delta_{y}^{\beta}\right) e^{k+2}\right\|+\left\|\left(\delta_{x}^{\alpha}+\delta_{y}^{\beta}\right) e^{k+1}\right\|+\left\|\left(\delta_{x}^{\alpha}+\delta_{y}^{\beta}\right) e^{k}\right\|
$$$$
\left.+\left\|\left(\delta_{x}^{\alpha}+\delta_{y}^{\beta}\right) e^{k-1}\right\|+\left\|\left(\delta_{x}^{\alpha}+\delta_{y}^{\beta}\right) e^{k-2}\right\|\right),
$$

$$
\begin{aligned}
\left\|J_{5}\right\|= & \left\|\Delta_{t}\left(U^{k}\right)^{*} e^{\bar{k}} e^{\hat{k}}+\Delta_{t} e^{k}\left(U^{\bar{k}}\right)^{*} e^{\hat{k}}+\left(U^{\bar{k}}\right)^{*} e^{\bar{k}} \Delta_{t} e^{\bar{k}}\right\| \\
\leqslant & \left\|u_{t}\right\|_{\infty} \cdot\left\|e^{\bar{k}}\right\| \cdot\left\|e^{\hat{k}}\right\|+\|u\|_{\infty} \cdot\left\|e^{\hat{k}}\right\| \cdot\left\|\Delta_{t} e^{k}\right\|+\|u\|_{\infty} \cdot\left\|e^{\bar{k}}\right\| \cdot\left\|\Delta_{t} e^{\bar{k}}\right\| \\
\leqslant & c_{7}\left(\left\|e^{\hat{k}}\right\|+\left\|\Delta_{t} e^{k}\right\|+\left\|\Delta_{t} e^{\bar{k}}\right\|\right) \\
\leqslant & c_{7}\left(\left\|e^{k+2}\right\|+\left\|e^{k+1}\right\|+\left\|e^{k}\right\|+\left\|e^{k-1}\right\|+\left\|e^{k-2}\right\|\right. \\
& +\left\|R^{k+1}\right\|+\left\|R^{k}\right\|+\left\|R^{k-1}\right\|+\left\|p^{k+1}\right\|+\left\|p^{k}\right\|+\left\|p^{k-1}\right\| \\
& +\left\|\left(\delta_{x}^{\alpha}+\delta_{y}^{\beta}\right) e^{k+2}\right\|+\left\|\left(\delta_{x}^{\alpha}+\delta_{y}^{\beta}\right) e^{k+1}\right\|+\left\|\left(\delta_{x}^{\alpha}+\delta_{y}^{\beta}\right) e^{k}\right\| \\
& \left.+\left\|\left(\delta_{x}^{\alpha}+\delta_{y}^{\beta}\right) e^{k-1}\right\|+\left\|\left(\delta_{x}^{\alpha}+\delta_{y}^{\beta}\right) e^{k-2}\right\|\right)
\end{aligned}
$$

$$
\begin{aligned}
\left\|J_{6}\right\| & =\left\|\Delta_{t} e^{k}\left(e^{\bar{k}}\right)^{*} U^{\hat{k}}+\Delta_{t}\left(e^{k}\right)^{*} e^{\bar{k}} U^{\hat{k}}+\Delta_{t} U^{\bar{k}} e^{\bar{k}}\left(e^{\bar{k}}\right)^{*}\right\| \\
& \leqslant 2\|u\|_{\infty} \cdot\left\|e^{\bar{k}}\right\| \cdot\left\|\Delta_{t} e^{k}\right\|+\frac{1}{2}\left\|u_{t}\right\|_{\infty} \cdot\left\|e^{\bar{k}}\right\| \cdot\left(\left\|e^{k+1}\right\|+\left\|e^{k-1}\right\|\right) \\
& \leqslant c_{7}\left(\left\|e^{k+1}\right\|+\left\|e^{k-1}\right\|+\left\|\Delta_{t} e^{k}\right\|\right) \\
& \leqslant c_{7}\left(\left\|e^{k+1}\right\|+\left\|e^{k-1}\right\|+\left\|R^{k}\right\|+\left\|p^{k}\right\|+\left\|\left(\delta_{x}^{\alpha}+\delta_{y}^{\beta}\right) e^{k+1}\right\|+\left\|\left(\delta_{x}^{\alpha}+\delta_{y}^{\beta}\right) e^{k-1}\right\|\right)
\end{aligned}
$$

$$
\begin{aligned}
\left\|J_{7}\right\|= & \left\|\Delta_{t} e^{k}\left(e^{\bar{k}}\right)^{*} e^{\hat{k}}+\Delta_{t}\left(e^{k}\right)^{*} e^{\bar{k}} e^{\hat{k}}+e^{\bar{k}}\left(e^{\bar{k}}\right)^{*} \Delta_{t} e^{\bar{k}}\right\| \\
\leqslant & 2\left\|e^{\bar{k}}\right\| \cdot\left\|e^{\hat{k}}\right\| \cdot\left\|\Delta_{t} e^{k}\right\|+\left\|e^{\bar{k}}\right\|^{2} \cdot\left\|\Delta_{t} e^{\bar{k}}\right\| \leqslant c_{7}\left(\left\|\Delta_{t} e^{k}\right\|+\left\|\Delta_{t} e^{\bar{k}}\right\|\right) \\
\leqslant & c_{7}\left(\left\|e^{k+2}\right\|+\left\|e^{k+1}\right\|+\left\|e^{k}\right\|+\left\|e^{k-1}\right\|+\left\|e^{k-2}\right\|+\left\|R^{k+1}\right\|+\left\|R^{k}\right\|+\left\|R^{k-1}\right\|\right. \\
& +\left\|p^{k+1}\right\|+\left\|p^{k}\right\|+\left\|p^{k-1}\right\|+\left\|\left(\delta_{x}^{\alpha}+\delta_{y}^{\beta}\right) e^{k+2}\right\|+\left\|\left(\delta_{x}^{\alpha}+\delta_{y}^{\beta}\right) e^{k+1}\right\| \\
& \left.+\left\|\left(\delta_{x}^{\alpha}+\delta_{y}^{\beta}\right) e^{k}\right\|+\left\|\left(\delta_{x}^{\alpha}+\delta_{y}^{\beta}\right) e^{k-1}\right\|+\left\|\left(\delta_{x}^{\alpha}+\delta_{y}^{\beta}\right) e^{k-2}\right\|\right) .
\end{aligned}
$$

Combining (3.33) and (3.44), we have

$$
\begin{aligned}
\left\|p^{k}\right\|^{2} & =\left\|\left|u^{k}\right|^{2} e^{\bar{k}}+U^{k} U^{\bar{k}}\left(e^{k}\right)^{*}+U^{\bar{k}}\left(u^{k}\right)^{*} e^{k}\right\|^{2} \\
& \leqslant\left\|\left(C_{u}+1\right)^{2} e^{\bar{k}}+C_{u}\left(2 C_{u}+1\right) e^{k}\right\|^{2} \\
& \leqslant 3 c_{8}^{2}\left(\left\|e^{k+1}\right\|^{2}+\left\|e^{k}\right\|^{2}+\left\|e^{k-1}\right\|^{2}\right) \leqslant 9 c_{8}^{2} c_{3}\left(\tau^{2}+h_{x}^{2}+h_{y}^{2}\right)^{2},
\end{aligned}
$$


Then combining with (3.74)-3.80), we have

$$
\begin{aligned}
& \left|\left(\Delta_{t} p^{k},\left(\delta_{x}^{\alpha}+\delta_{y}^{\beta}\right) e^{k}\right)\right| \leqslant \sum_{i=1}^{7}\left\|J_{i}\right\| \cdot\left\|\left(\delta_{x}^{\alpha}+\delta_{y}^{\beta}\right) e^{k}\right\| \\
\leqslant & c_{7}\left(\left\|e^{k+2}\right\|^{2}+\left\|e^{k+1}\right\|^{2}+\left\|e^{k}\right\|^{2}+\left\|e^{k-1}\right\|^{2}+\left\|e^{k-2}\right\|^{2}\right) \\
& +c_{7}\left(\left\|\left(\delta_{x}^{\alpha}+\delta_{y}^{\beta}\right) e^{k+2}\right\|^{2}+\left\|\left(\delta_{x}^{\alpha}+\delta_{y}^{\beta}\right) e^{k+1}\right\|^{2}+\left\|\left(\delta_{x}^{\alpha}+\delta_{y}^{\beta}\right) e^{k}\right\|^{2}\right. \\
& \left.+\left\|\left(\delta_{x}^{\alpha}+\delta_{y}^{\beta}\right) e^{k-1}\right\|^{2}+\left\|\left(\delta_{x}^{\alpha}+\delta_{y}^{\beta}\right) e^{k-2}\right\|^{2}\right) \\
& +c_{7}\left(\left\|R^{k+1}\right\|^{2}+\left\|R^{k}\right\|^{2}+\left\|R^{k-1}\right\|^{2}+\left\|p^{k+1}\right\|^{2}+\left\|p^{k}\right\|^{2}+\left\|p^{k-1}\right\|^{2}\right) .
\end{aligned}
$$

According to 3.81)-3.82, we have

$$
\begin{aligned}
& \frac{8 \tau}{\nu} \sum_{k=1}^{n} \operatorname{Re}\left\{(\kappa+\mathbf{i} \zeta)\left|\left(p^{k},\left(\delta_{x}^{\alpha}+\delta_{y}^{\beta}\right) \Delta_{t} e^{k}\right)\right|\right\} \\
\leqslant & \frac{8 \sqrt{\kappa^{2}+\zeta^{2}}}{\nu} \tau \sum_{k=2}^{n-1}\left\|\Delta_{t} p^{k}\right\| \cdot\left\|\left(\delta_{x}^{\alpha}+\delta_{y}^{\beta}\right) e^{k}\right\|+\frac{4 \sqrt{\kappa^{2}+\zeta^{2}}}{\nu}\left[\left\|p^{n}\right\| \cdot\left\|\left(\delta_{x}^{\alpha}+\delta_{y}^{\beta}\right) e^{n+1}\right\|\right. \\
& \left.+\left\|p^{n-1}\right\| \cdot\left\|\left(\delta_{x}^{\alpha}+\delta_{y}^{\beta}\right) e^{n}\right\|+\left\|p^{2}\right\| \cdot\left\|\left(\delta_{x}^{\alpha}+\delta_{y}^{\beta}\right) e^{1}\right\|\right] \\
\leqslant & \frac{8 \sqrt{\kappa^{2}+\zeta^{2}}}{\nu} \tau \sum_{k=2}^{n-1}\left(\sum_{i=1}^{7}\left\|J_{i}\right\| \cdot\left\|\left(\delta_{x}^{\alpha}+\delta_{y}^{\beta}\right) e^{k}\right\|\right)+4\left(\frac{4\left(\kappa^{2}+\zeta^{2}\right)}{\nu^{2}}\left\|p^{n}\right\|^{2}+\frac{1}{16}\left\|\left(\delta_{x}^{\alpha}+\delta_{y}^{\beta}\right) e^{n+1}\right\|^{2}\right. \\
& \left.+\frac{4\left(\kappa^{2}+\zeta^{2}\right)}{\nu^{2}}\left\|p^{n-1}\right\|^{2}+\frac{1}{16}\left\|\left(\delta_{x}^{\alpha}+\delta_{y}^{\beta}\right) e^{n}\right\|^{2}+\frac{\kappa^{2}+\zeta^{2}}{2 \nu^{2}}\left\|p^{2}\right\|^{2}+\frac{1}{2}\left\|\left(\delta_{x}^{\alpha}+\delta_{y}^{\beta}\right) e^{1}\right\|^{2}\right) \\
\leqslant & \frac{8 \sqrt{\kappa^{2}+\zeta^{2}}}{\nu} \tau \sum_{k=2}^{n-1} c_{7}\left(\left\|e^{k+2}\right\|^{2}+\left\|e^{k+1}\right\|^{2}+\left\|e^{k}\right\|^{2}+\left\|e^{k-1}\right\|^{2}+\left\|e^{k-2}\right\|^{2}\right) \\
& +\frac{8 \sqrt{\kappa^{2}+\zeta^{2}}}{\nu} \tau \sum_{k=2}^{n-1} c_{7}\left(\left\|\left(\delta_{x}^{\alpha}+\delta_{y}^{\beta}\right) e^{k+2}\right\|^{2}+\left\|\left(\delta_{x}^{\alpha}+\delta_{y}^{\beta}\right) e^{k+1}\right\|^{2}+\left\|\left(\delta_{x}^{\alpha}+\delta_{y}^{\beta}\right) e^{k}\right\|^{2}\right. \\
& \left.+\left\|\left(\delta_{x}^{\alpha}+\delta_{y}^{\beta}\right) e^{k-1}\right\|^{2}+\left\|\left(\delta_{x}^{\alpha}+\delta_{y}^{\beta}\right) e^{k-2}\right\|^{2}\right) \\
& +\frac{8 \sqrt{\kappa^{2}+\zeta^{2}}}{\nu} \tau \sum_{k=2}^{n-1} c_{7}\left(\left\|R^{k+1}\right\|^{2}+\left\|R^{k}\right\|^{2}+\left\|R^{k-1}\right\|^{2}+\left\|p^{k+1}\right\|^{2}+\left\|p^{k}\right\|^{2}+\left\|p^{k-1}\right\|^{2}\right) \\
& +\frac{16\left(\kappa^{2}+\zeta^{2}\right)}{\nu^{2}}\left\|p^{n}\right\|^{2}+\frac{16\left(\kappa^{2}+\zeta^{2}\right)}{\nu^{2}}\left\|p^{n-1}\right\|^{2}+\frac{1}{4}\left\|\left(\delta_{x}^{\alpha}+\delta_{y}^{\beta}\right) e^{n+1}\right\|^{2}+\frac{1}{4}\left\|\left(\delta_{x}^{\alpha}+\delta_{y}^{\beta}\right) e^{n}\right\|^{2} \\
& +\frac{2\left(\kappa^{2}+\zeta^{2}\right)}{\nu^{2}}\left\|p^{2}\right\|^{2}+2\left\|\left(\delta_{x}^{\alpha}+\delta_{y}^{\beta}\right) e^{1}\right\|^{2} \\
\leqslant & 40 c_{9} \tau \sum_{k=1}^{n}\left\|\left(\delta_{x}^{\alpha}+\delta_{y}^{\beta}\right) e^{k}\right\|^{2}+\left(8 c_{9} \tau+\frac{1}{4}\right)\left(\left\|\left(\delta_{x}^{\alpha}+\delta_{y}^{\beta}\right) e^{n+1}\right\|^{2}+\left\|\left(\delta_{x}^{\alpha}+\delta_{y}^{\beta}\right) e^{n}\right\|^{2}\right) \\
& +c_{10}\left(\tau^{2}+h_{x}^{2}+h_{y}^{2}\right)^{2} .
\end{aligned}
$$

Substituting (3.68), (3.70) and (3.83) into (3.67), we have

$$
F_{n} \leqslant 16 c_{4} \tau \sum_{k=1}^{n} F_{k-1}+F_{0}+\left(40 c_{9}+4\right) \tau \sum_{k=1}^{n}\left\|\left(\delta_{x}^{\alpha}+\delta_{y}^{\beta}\right) e^{k}\right\|^{2}
$$




$$
\begin{aligned}
& +\left(8 c_{9} \tau+\frac{1}{2}\right)\left(\left\|\left(\delta_{x}^{\alpha}+\delta_{y}^{\beta}\right) e^{n+1}\right\|^{2}+\left\|\left(\delta_{x}^{\alpha}+\delta_{y}^{\beta}\right) e^{n}\right\|^{2}\right)+\left(c_{5}+c_{6}+c_{7}\right)\left(\tau^{2}+h_{x}^{2}+h_{y}^{2}\right)^{2} \\
\leqslant & \left(40 c_{9}+16 c_{4}+4\right) \tau \sum_{k=1}^{n} F_{k-1}+\left(8 c_{9} \tau+\frac{1}{2}\right)\left(\left\|\left(\delta_{x}^{\alpha}+\delta_{y}^{\beta}\right) e^{n+1}\right\|^{2}+\left\|\left(\delta_{x}^{\alpha}+\delta_{y}^{\beta}\right) e^{n}\right\|^{2}\right) \\
& +\left(c_{5}+c_{6}+c_{7}\right)\left(\tau^{2}+h_{x}^{2}+h_{y}^{2}\right)^{2} .
\end{aligned}
$$

When $\frac{1}{2}-8 c_{9} \tau \geqslant \frac{1}{4}$, we have

$$
F_{n} \leqslant 4\left(40 c_{9}+16 c_{4}+4\right) \tau \sum_{k=1}^{n} F_{k-1}+4 F_{0}+4\left(c_{5}+c_{6}+c_{7}\right)\left(\tau^{2}+h_{x}^{2}+h_{y}^{2}\right)^{2} .
$$

With the help of (3.39) and (3.42), we have

$$
F_{0}=\left\|\left(\delta_{x}^{\alpha}+\delta_{y}^{\beta}\right) e^{1}\right\|^{2}+\left\|\Lambda^{\alpha, \beta} e^{1}\right\|^{2} \leqslant\left(\frac{4}{\nu^{2}}+\frac{4}{|\gamma| \nu}\right) C_{R}^{2}|\Omega|\left(\tau^{2}+h_{x}^{2}+h_{y}^{2}\right)^{2},
$$

and (3.85) becomes

$$
\begin{aligned}
F_{n} \leqslant & \left(160 c_{9}+64 c_{4}+16\right) \tau \sum_{k=1}^{n} F_{k-1} \\
& +\left[\left(\frac{4}{\nu^{2}}+\frac{4}{|\gamma| \nu}\right) C_{R}^{2}|\Omega|+4\left(c_{5}+c_{6}+c_{7}\right)\right]\left(\tau^{2}+h_{x}^{2}+h_{y}^{2}\right)^{2} .
\end{aligned}
$$

By the discrete Gronwall's inequality, we have

$$
\begin{aligned}
F_{n} \leqslant & {\left[\left(\frac{4}{\nu^{2}}+\frac{4}{|\gamma| \nu}\right) C_{R}^{2}|\Omega|+4\left(c_{5}+c_{6}+c_{7}\right)\right] } \\
& \times \exp \left\{\sum_{k=1}^{n}\left(160 c_{9}+64 c_{4}+16\right) \tau\right\}\left(\tau^{2}+h_{x}^{2}+h_{y}^{2}\right)^{2} \\
\leqslant & {\left[\left(\frac{4}{\nu^{2}}+\frac{4}{|\gamma| \nu}\right) C_{R}^{2}|\Omega|+4\left(c_{5}+c_{6}+c_{7}\right)\right] \exp \left(\left(160 c_{9}+64 c_{4}+16\right) T\right)\left(\tau^{2}+h_{x}^{2}+h_{y}^{2}\right)^{2} } \\
= & c_{11}\left(\tau^{2}+h_{x}^{2}+h_{y}^{2}\right)^{2} .
\end{aligned}
$$

We now combine the results (3.55) and (3.88), to recover

$$
\begin{aligned}
& \left\|e^{n+1}\right\|^{2}+\left\|\Lambda^{\alpha, \beta} e^{n+1}\right\|^{2}+\left\|\left(\delta_{x}^{\alpha}+\delta_{y}^{\beta}\right) e^{n+1}\right\|^{2} \\
\leqslant & E_{n}+F_{n} \leqslant\left(c_{3}+c_{11}\right)\left(\tau^{2}+h_{x}^{2}+h_{y}^{2}\right)^{2}, \quad 0 \leqslant n \leqslant l .
\end{aligned}
$$

Using the Sobolev imbedding theorem in Lemma 2.1 we have

$$
\begin{aligned}
\left\|e^{n+1}\right\|_{l^{\infty}}^{2} & \leqslant \frac{\left(C_{\alpha, \beta}\right)^{2}}{\left(\widetilde{C}_{\alpha, \beta}\right)^{2}}\left(\left\|e^{n+1}\right\|^{2}+\left\|\Lambda^{\alpha, \beta} e^{n+1}\right\|^{2}+\left\|\left(\delta_{x}^{\alpha}+\delta_{y}^{\beta}\right) e^{n+1}\right\|^{2}\right) \\
& :=c_{12}^{2}\left(\tau^{2}+h_{x}^{2}+h_{y}^{2}\right)^{2}, \quad 0 \leqslant n \leqslant l .
\end{aligned}
$$

Consequently,

$$
\left\|e^{n}\right\|_{l \infty} \leqslant c_{12}\left(\tau^{2}+h_{x}^{2}+h_{y}^{2}\right), \quad 0 \leqslant n \leqslant N .
$$

This completes the proof. 
Remark 3.2 Although the theoretical results were proved on the requirement of the temporal step sizes in (3.37), (3.42), (3.53), (3.66), 3.85), the numerical examples in Section 4 show that these conditions might be relaxed.

Corollary 1 (Stability) Let $u(x, y, t)$ be the solution of the problem (3.1)-(3.3) and $\left\{u_{i j}^{n} \mid(i, j) \in\right.$ $\bar{\omega}, 0 \leqslant n \leqslant N\}$ be the solution of the scheme [3.18] - (3.21]. Then for $\tau \leqslant \tau_{0}$ and $\max \left\{h_{x}, h_{y}\right\} \leqslant$ $h_{0}$, we have

$$
\left\|u^{n}\right\|_{l^{\infty}} \leqslant c_{13}, \quad 0 \leqslant n \leqslant N
$$

where $\tau_{0}$ and $h_{0}$ are some positive constants.

Proof From Theorem 3.2, we have

$$
\left\|u^{n}\right\|_{l^{\infty}}=\left\|U^{n}-e^{n}\right\|_{l^{\infty}} \leqslant\left\|U^{n}\right\|_{l^{\infty}}+\left\|e^{n}\right\|_{l^{\infty}} \leqslant C_{u}+c_{13}\left(\tau_{0}^{2}+2 h_{0}^{2}\right), \quad 0 \leqslant n \leqslant N,
$$

for $\tau \leqslant \tau_{0}$ and $h_{x}, h_{y} \leqslant h_{0}$. When $\tau_{0}^{2}+2 h_{0}^{2} \leqslant \frac{1}{c_{13}}$, we have

$$
\left\|u^{n}\right\|_{l \infty} \leqslant c_{13}, \quad 0 \leqslant n \leqslant N,
$$

where $c_{13}=C_{u}+1$. This completes the proof.

\section{Numerical validations}

We present two examples to verify stability and convergence in the pointwise sense with order of convergence $\mathcal{O}\left(\tau^{2}+h_{x}^{2}+h_{y}^{2}\right)$.

To improve the computational efficiency, we use the preconditioned GMRES algorithm to solve the linear system of equations which arises from the numerical scheme (3.18)-(3.21). The computational cost of applying the linear system of equations can be reduced from $\left(M_{x}^{3} M_{y}^{3}\right)$ to $\left(M_{x} M_{y}\left(\log M_{x}+\log M_{y}\right)\right)$ with the help of the two-dimensional fast Fourier transform (FFT). The detailed illustration on the preconditioner and the algorithm is discussed in [40]. The stopping criterion of GMRES is $\left\|r^{k}\right\| /\left\|r^{0}\right\|<10^{-7}$, where $r^{k}$ denotes the residual vector of the complex linear system of equations after $k$ iterations, and the initial guess is chosen as the zero vector.

All numerical tests were performed on a Windows 10, 64 bit PC-Intel(R) Core(TM) i7-7500 CPU @ 2.70GHz, 4GB of RAM using MATLAB R2016a with the machine epsilon $10^{-16}$ in double precision floating point arithmetic.

The spatial step sizes along the $x$ - and $y$-directions are taken to be equal $\left(h_{x}=h_{y}=h\right.$ or $\left.M_{x}=M_{y}=M\right)$. We define the discrete $l^{\infty}$-norm for the numerical error as follows

$$
\|E(h, \tau)\|_{l^{\infty}}=\max _{1 \leqslant i, j \leqslant M}\left\{\left|U_{i j}^{N}-u_{i j}^{N}\right|\right\} .
$$

- When the exact solution is known, we will test the order of convergence in space and time, respectively, by

$$
\operatorname{Ord}_{l^{\infty}}^{h}=\log _{2}\left(\frac{\|E(h, \tau)\|_{l^{\infty}}}{\|E(h / 2, \tau)\|_{l^{\infty}}}\right), \quad \operatorname{Ord}_{l^{\infty}}^{\tau}=\log _{2}\left(\frac{\|E(h, \tau)\|_{l^{\infty}}}{\|E(h, \tau / 2)\|_{l^{\infty}}}\right) .
$$


- When the exact solution is unknown, we can test the order of convergence in space and time, respectively, by the following posterior error estimation

$$
\operatorname{Ord}_{l^{\infty}}^{h}=\log _{2} \frac{\|u(h, \tau)-u(h / 2, \tau)\|_{l^{\infty}}}{\|u(h / 2, \tau)-u(h / 4, \tau)\|_{l^{\infty}}}, \quad \operatorname{Ord}_{l^{\infty}}^{\tau}=\log _{2} \frac{\|u(h, \tau)-u(h, \tau / 2)\|_{l^{\infty}}}{\|u(h, \tau / 2)-u(h, \tau / 4)\|_{l^{\infty}}} .
$$

Example 1 Consider the problem in 40,

$$
\begin{gathered}
\partial_{t} u-(\nu+\mathbf{i} \eta)\left(\partial_{x}^{\alpha} u+\partial_{y}^{\beta} u\right)+(\kappa+\mathbf{i} \zeta)|u|^{2} u-\gamma u=f(x, y, t), \\
(x, y) \in(-1,1) \times(-1,1), t \in(0,1],
\end{gathered}
$$

where $\nu=\eta=\kappa=1, \zeta=2, \gamma=3$ and

$$
\begin{aligned}
f(x, y, t)= & -\mathbf{i} \exp (-\mathbf{i} t) \cdot(1+x)^{4}(1-x)^{4}(1+y)^{4}(1-y)^{4} \\
& +(\nu+\eta \mathbf{i})(1+y)^{4}(1-y)^{4} \frac{\exp (-\mathbf{i} t)}{2 \cos \frac{\alpha \pi}{2}} \\
& \times\left\{\sum_{k=0}^{4}(-1)^{k}\left(\begin{array}{l}
4 \\
k
\end{array}\right) 2^{4-k} \frac{\Gamma(k+5)}{\Gamma(k+5-\alpha)}\left[(1+x)^{k+4-\alpha}+(1-x)^{k+4-\alpha}\right]\right\} \\
& +(\nu+\eta \mathbf{i})(1+x)^{4}(1-x)^{4} \frac{\exp (-\mathbf{i} t)}{2 \cos \frac{\beta \pi}{2}} \\
& \times\left\{\sum_{k=0}^{4}(-1)^{k}\left(\begin{array}{l}
4 \\
k
\end{array}\right) 2^{4-k} \frac{\Gamma(k+5)}{\Gamma(k+5-\beta)}\left[(1+x)^{k+4-\beta}+(1-x)^{k+4-\beta}\right]\right\} \\
& +(\kappa+\eta \mathbf{i})\left|(1+x)^{4}(1-x)^{4}(1+y)^{4}(1-y)^{4} \exp (-\mathbf{i} t)\right|^{2} \\
& \times(1+x)^{4}(1-x)^{4}(1+y)^{4}(1-y)^{4} \cdot \exp (-\mathbf{i} t) \\
& -\gamma(1+x)^{4}(1-x)^{4}(1+y)^{4}(1-y)^{4} \cdot \exp (-\mathbf{i} t) .
\end{aligned}
$$

The initial-boundary value conditions of the above truncated problem are determined by the exact solution $u(x, y, t)=(x+1)^{4}(x-1)^{4}(y+1)^{4}(y-1)^{4} \exp (-\mathbf{i} t)$.

Numerical results are reported in Tables 1, 2 and Figures 12.

Table 1 shows that the order of convergence (calculated by (4.1) ) in the spatial dimension in the pointwise sense are in perfect agreement with the theoretical results for different fractional orders $(\alpha, \beta)$. The temporal orders of convergence (calculated by (4.1)) in the pointwise sense are shown in Table 2 which confirm that our numerical scheme are secondorder accurate independent of the values we take for the fractional orders $\alpha$ and $\beta$.

Figure 1 shows that the numerical errors at the fixed the time step but varying space step size $(M=8,16,32,64,128,256)$ with different fractional orders $\alpha$ and $\beta$. We see clearly that the numerical errors ultimately approach a fixed value as the spatial error dominates the temporal error. This illustrates that our numerical algorithm does not require a timestep restriction, which indirectly confirms that the stability for the current algorithm is very good.

The numerical solutions and corresponding contour profiles are illustrated in Figure 2 for different fractional orders $\alpha$ and $\beta$ at the same final time. From these figures, we see that the fractional orders directly affect the numerical results. For example, when $(\alpha, \beta)=(1.2,1.8)$, the problem will be anisotropic as captured by the numerical result. 


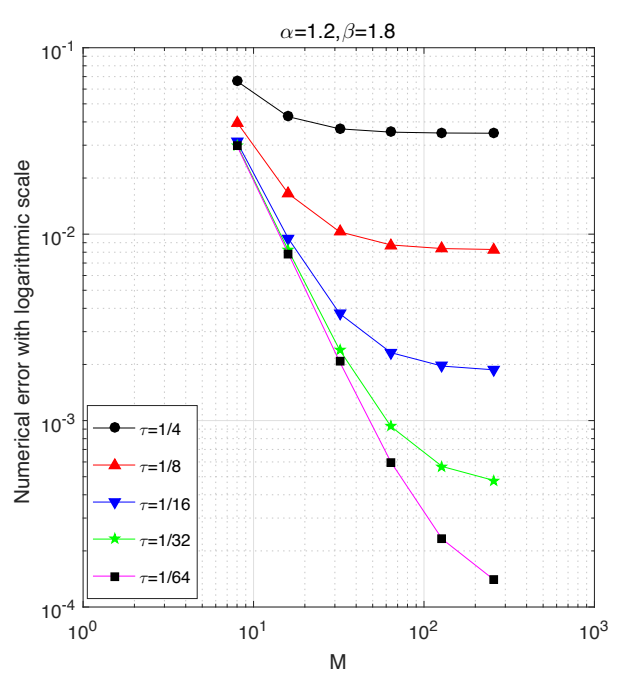

(a)

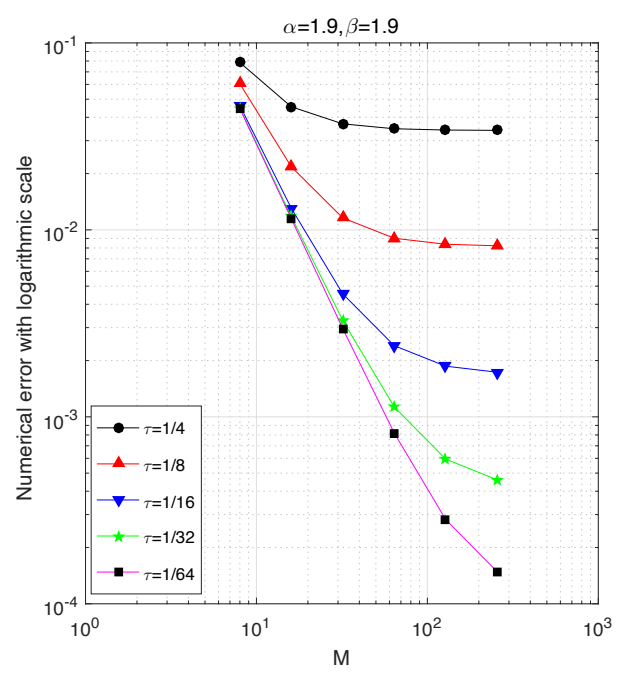

(b)

Fig. 1 The numerical errors in logarithmic scale via the fixed $\tau$ but varying $h$ for the stability test with different $\alpha$ and $\beta$
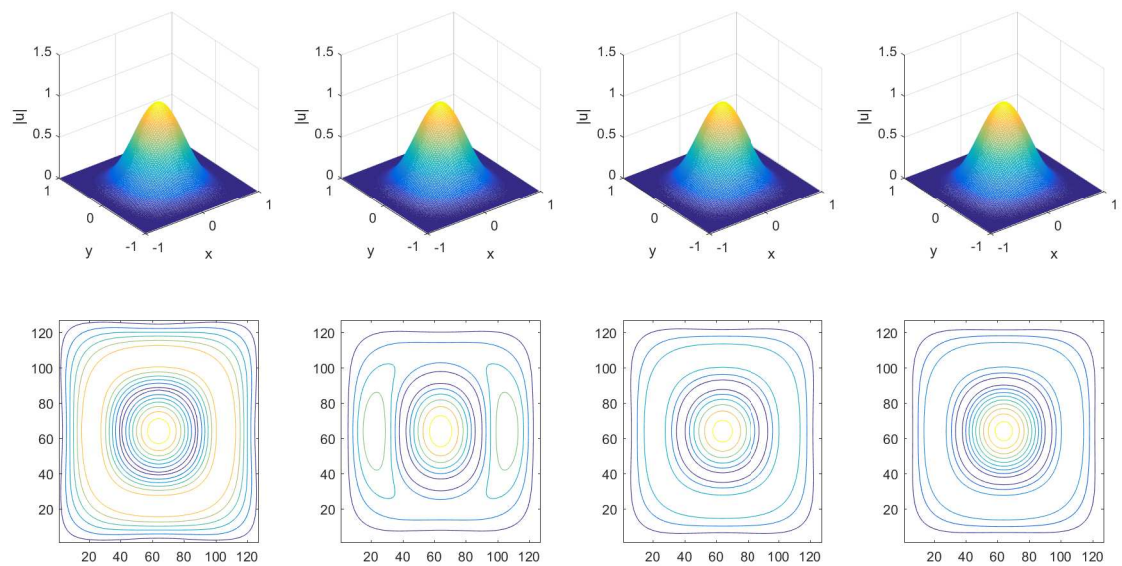

Fig. 2 The numerical solutions and corresponding contour profiles. The parameters are taken with $(\alpha, \beta)=$ $(1.1,1.1),(1.2,1.8),(1.5,1.5),(1.9,1.9)$ from left to right; The final time is $T=1$ and the grid sizes $M=128$, $N=200$ 
Table 1 The maximum norm errors versus temporal grid size reduction and convergence orders of the difference scheme (3.18) - 3.21) in time with fixed $h=1 / 256$

\begin{tabular}{|c|c|c|c|}
\hline$(\alpha, \beta)$ & $\tau$ & $\|E(h, \tau)\|_{l \infty}$ & $\operatorname{Ord}_{l \infty}^{\tau}$ \\
\hline \multirow{3}{*}{$(1.2,1.8)$} & $1 / 2$ & $1.9363 \mathrm{e}-1$ & $*$ \\
& $1 / 4$ & $3.4756 \mathrm{e}-2$ & 2.4780 \\
& $1 / 8$ & $8.2517 \mathrm{e}-3$ & 2.0745 \\
& $1 / 16$ & $1.8508 \mathrm{e}-3$ & 2.1565 \\
& $1 / 32$ & $4.5568 \mathrm{e}-4$ & 2.0220 \\
\hline \multirow{3}{*}{$(1.5,1.5)$} & $1 / 2$ & $1.9339 \mathrm{e}-1$ & $*$ \\
& $1 / 4$ & $3.4412 \mathrm{e}-2$ & 2.4905 \\
& $1 / 8$ & $8.6226 \mathrm{e}-3$ & 1.9967 \\
& $1 / 16$ & $1.9118 \mathrm{e}-3$ & 2.1732 \\
& $1 / 32$ & $4.6982 \mathrm{e}-4$ & 2.0247 \\
\hline \multirow{3}{*}{$(1.9,1.1)$} & $1 / 2$ & $1.9385 \mathrm{e}-1$ & 2.4708 \\
& $1 / 4$ & $3.4969 \mathrm{e}-2$ & 2.1258 \\
& $1 / 8$ & $8.0120 \mathrm{e}-3$ & 2.1573 \\
& $1 / 16$ & $1.7961 \mathrm{e}-3$ & 2.0148 \\
\hline
\end{tabular}

Table 2 The maximum norm errors versus spatial grid size reduction and convergence orders of the difference scheme (3.18) -3.21 in space with fixed $\tau=1 / 512$

\begin{tabular}{|c|c|c|c|}
\hline$(\alpha, \beta)$ & $h$ & $\|E(h, \tau)\|_{l^{\infty}}$ & Ord $_{l^{\infty}}^{h}$ \\
\hline \multirow{3}{*}{$(1.2,1.8)$} & $1 / 4$ & $2.9701 \mathrm{e}-2$ & $*$ \\
& $1 / 8$ & $7.7677 \mathrm{e}-3$ & 1.9350 \\
& $1 / 16$ & $1.9633 \mathrm{e}-3$ & 1.9842 \\
& $1 / 32$ & $4.9292 \mathrm{e}-4$ & 1.9939 \\
& $1 / 64$ & $1.2444 \mathrm{e}-4$ & 1.9859 \\
\hline \multirow{3}{*}{$(1.5,1.5)$} & $1 / 4$ & $2.8914 \mathrm{e}-2$ & $*$ \\
& $1 / 8$ & $7.4816 \mathrm{e}-3$ & 1.9504 \\
& $1 / 16$ & $1.8818 \mathrm{e}-3$ & 1.9912 \\
& $1 / 32$ & $4.7148 \mathrm{e}-4$ & 1.9968 \\
& $1 / 64$ & $1.1902 \mathrm{e}-4$ & 1.9860 \\
\hline \multirow{3}{*}{$(1.9,1.1)$} & $1 / 4$ & $3.0190 \mathrm{e}-2$ & $*$ \\
& $1 / 8$ & $7.9503 \mathrm{e}-3$ & 1.9250 \\
& $1 / 16$ & $2.0157 \mathrm{e}-3$ & 1.9797 \\
& $1 / 32$ & $5.0667 \mathrm{e}-4$ & 1.9922 \\
& $1 / 64$ & $1.2793 \mathrm{e}-4$ & 1.9856 \\
\hline
\end{tabular}

Example 2 Consider the nonlinear fractional Ginzburg-Landau equation as

$$
\begin{aligned}
& \partial_{t} u-(\nu+\mathbf{i} \eta)\left(\partial_{x}^{\alpha} u+\partial_{y}^{\beta} u\right)+(\kappa+\mathbf{i} \zeta)|u|^{2} u-\gamma u=0, \quad(x, y) \in \Omega, t \in(0, T], \\
& u(x, y, t)=0, \quad(x, y) \in \partial \Omega, t \in(0, T], \\
& u(x, y, 0)=\operatorname{sech}(x) \operatorname{sech}(y) \exp (\mathbf{i}(x+y)), \quad(x, y) \in \bar{\Omega}=\Omega \cup \partial \Omega,
\end{aligned}
$$

where $\Omega=(-10,10) \times(-10,10), \nu=\eta=\kappa=\zeta=\gamma=1$. The exact solution is unknown.

The convergence behaviors of the proposed algorithm are reported in Tables 3 S Since the exact solution is unknown, we calculate orders of convergence by (4.2). Similar results 
are observed as for Example 1 which show that our theoretical result is consistent with the numerical results in the pointwise sense.

In addition, we study the numerical evolution in Figure 3 and the influence of the fractional orders in Figure 4 . In Figure 3 for fixed $\alpha$ and $\beta$, we see that the numerical results are diffusive. In Figure 4 we find that the fractional orders indeed have an effect on the solution.

Table 3 The maximum norm errors versus temporal grid size reduction and convergence orders of the difference scheme (3.18)-(3.21) in time with fixed $h=5 / 64, T=1$

\begin{tabular}{|c|c|c|c|}
\hline$(\alpha, \beta)$ & $\tau$ & $\|E(h, \tau)\|_{l \infty}$ & $\operatorname{Ord}_{l_{\infty}}^{\tau}$ \\
\hline \multirow{3}{*}{$(1.1,1.9)$} & $1 / 4$ & $1.1760 \mathrm{e}-2$ & $*$ \\
& $1 / 8$ & $2.7348 \mathrm{e}-3$ & 2.1044 \\
& $1 / 16$ & $6.4542 \mathrm{e}-4$ & 2.0831 \\
& $1 / 32$ & $1.5920 \mathrm{e}-4$ & 2.0194 \\
& $1 / 64$ & $3.9065 \mathrm{e}-5$ & 2.0269 \\
\hline \multirow{3}{*}{$(1.5,1.5)$} & $1 / 4$ & $9.7617 \mathrm{e}-3$ & $*$ \\
& $1 / 8$ & $2.3333 \mathrm{e}-3$ & 2.0647 \\
& $1 / 16$ & $5.6950 \mathrm{e}-4$ & 2.0346 \\
& $1 / 32$ & $1.4103 \mathrm{e}-4$ & 2.0137 \\
& $1 / 64$ & $3.5390 \mathrm{e}-5$ & 1.9946 \\
\hline \multirow{3}{*}{$(1.7,1.3)$} & $1 / 4$ & $1.0267 \mathrm{e}-2$ & $*$ \\
& $1 / 8$ & $2.4244 \mathrm{e}-3$ & 2.0823 \\
& $1 / 16$ & $5.8758 \mathrm{e}-4$ & 2.0448 \\
& $1 / 32$ & $1.4563 \mathrm{e}-4$ & 2.0125 \\
& $1 / 64$ & $3.6091 \mathrm{e}-5$ & 2.0126 \\
\hline
\end{tabular}

Table 4 The maximum norm errors versus spatial grid size reduction and convergence orders of the difference scheme 3.18 - 3.21 in space with fixed $\tau=1 / 16, T=1$

\begin{tabular}{|r|c|c|c|}
\hline$(\alpha, \beta)$ & $h$ & $\|E(h, \tau)\|_{l^{\infty}}$ & $\operatorname{Ord}_{l^{\infty}}^{h}$ \\
\hline \multirow{3}{*}{$(1.1,1.9)$} & $1 / 4$ & $1.0814 \mathrm{e}-2$ & $*$ \\
& $1 / 8$ & $2.7407 \mathrm{e}-3$ & 1.9803 \\
& $1 / 16$ & $6.8252 \mathrm{e}-4$ & 2.0056 \\
& $1 / 32$ & $1.7081 \mathrm{e}-4$ & 1.9985 \\
& $1 / 64$ & $4.2711 \mathrm{e}-5$ & 1.9997 \\
\hline \multirow{3}{*}{$(1.5,1.5)$} & $1 / 4$ & $1.0396 \mathrm{e}-2$ & $*$ \\
& $1 / 8$ & $2.6967 \mathrm{e}-3$ & 1.9468 \\
& $1 / 16$ & $6.7326 \mathrm{e}-4$ & 2.0020 \\
& $1 / 32$ & $1.6831 \mathrm{e}-4$ & 2.0001 \\
& $1 / 64$ & $4.2093 \mathrm{e}-5$ & 1.9994 \\
\hline \multirow{3}{*}{$(1.7,1.3)$} & $1 / 4$ & $1.0369 \mathrm{e}-2$ & $*$ \\
& $1 / 8$ & $2.6864 \mathrm{e}-3$ & 1.9485 \\
& $1 / 16$ & $6.7586 \mathrm{e}-4$ & 2.9909 \\
& $1 / 32$ & $1.6888 \mathrm{e}-4$ & 1.9995 \\
\hline
\end{tabular}



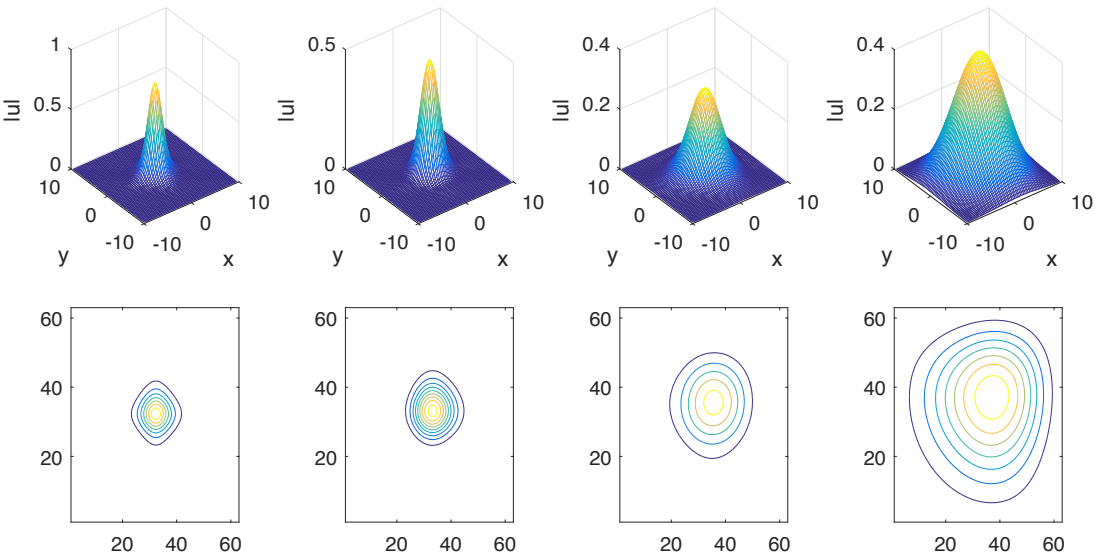

Fig. 3 The numerical solutions and corresponding contour profiles. The parameters are taken with $T=0.1$, $0.4,1.6,3.2$ from left to right and the grid sizes $\tau=0.01, h=5 / 16, \alpha=\beta=1.5$
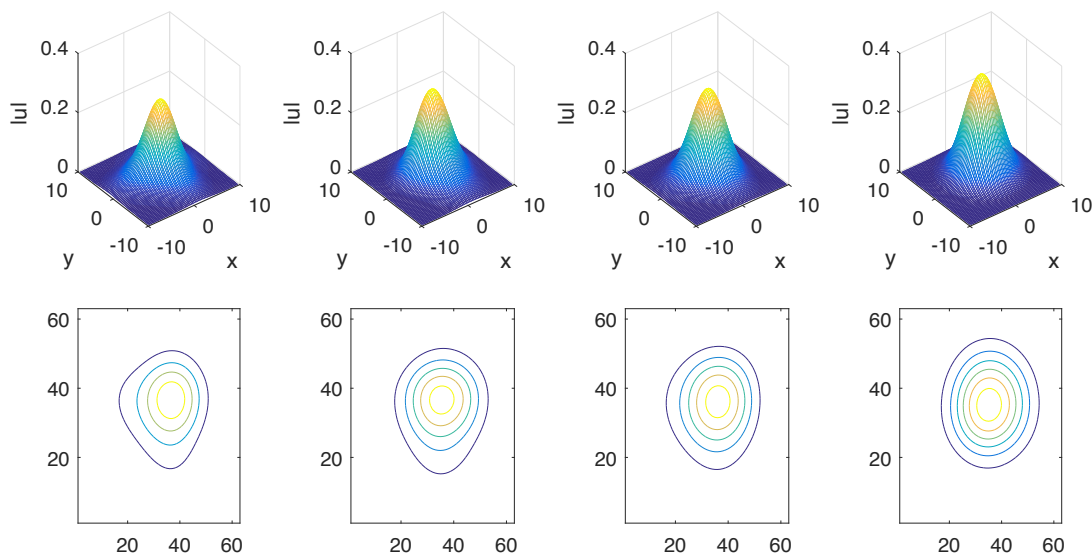

Fig. 4 The numerical solutions at the fixed time $T=2$ and corresponding contour profiles. The parameters are taken with $(\alpha, \beta)=(1.1,1.1),(1.2,1.8),(1.5,1.5),(1.9,1.9)$ from left to right and the grid sizes $M=64$, $N=100$

\section{Conclusion}

In this paper, we develop pointwise error estimates for a three-level linearized finite difference scheme for the two-dimensional nonlinear space fractional Ginzburg-Landau equation. The 
optimal error estimate $\mathcal{O}\left(\tau^{2}+h_{x}^{2}+h_{y}^{2}\right)$ is obtained in the discrete infinity norm. Solution uniqueness and stability under very mild conditions are also given. The theoretical results are confirmed by our numerical experiments. Moreover, the practical numerical implementation is efficient since the coefficient matrix, arising from the numerical scheme is a block Toeplitz matrix with a Toeplitz block, which can be efficiently preconditioned with the block circulant preconditioner and implemented by the two-dimensional FFT. The analysis technique can be extended to other two-dimensional problems with Riesz fractional derivative including the two-dimensional space-fractional diffusion equation [37] and the space-fractional Schrödinger equation [41]. In the future, we will study the pointwise error estimate of the higher order numerical scheme for the nonlinear space fractional Ginzburg-Landau equation in higher dimensions.

\section{Appendix}

The following lemma plays an important role in deriving the local truncation errors $R_{i j}^{n}$ and $\Delta_{t} R_{i j}$.

Lemma A.7 Let $g(t) \in C^{3}\left(\left[t_{n-1}, t_{n+1}\right]\right)$; then

$$
\begin{aligned}
& \frac{g\left(t_{n+1}\right)-g\left(t_{n-1}\right)}{2 \tau}=g^{\prime}\left(t_{n}\right)+\frac{\tau^{2}}{4} \int_{0}^{1}\left[g^{(3)}\left(t_{n}+s \tau\right)+g^{(3)}\left(t_{n}-s \tau\right)\right](1-s)^{2} d s, \\
& \frac{g\left(t_{n+1}\right)+g\left(t_{n-1}\right)}{2}=g\left(t_{n}\right)+\frac{\tau^{2}}{2} \int_{0}^{1}\left[g^{(2)}\left(t_{n}+s \tau\right)+g^{(2)}\left(t_{n}-s \tau\right)\right](1-s) d s .
\end{aligned}
$$

Proof Based on the Taylor expansion with the integral remainder

$$
g\left(t_{n}+\tau\right)=\sum_{i=0}^{k} \frac{\tau^{i}}{i !} g^{(i)}\left(t_{n}\right)+\frac{\tau^{k+1}}{k !} \int_{0}^{1} g^{(k+1)}\left(t_{n}+s \tau\right)(1-s)^{k} d s, \quad g \in C^{k+1},
$$

the results follow.

Lemma A.8 Suppose $\partial_{t} q\left(x, t_{n}\right) \in \mathscr{C}^{2+\alpha}(\mathbb{R})$. Then it holds

$$
\left(-h_{x}^{-\alpha} \delta_{x}^{\alpha} \Delta_{t} q\left(x, t_{n}\right)\right)-\partial_{x}^{\alpha} \Delta_{t} q\left(x, t_{n}\right)=\mathcal{O}\left(h_{x}^{2}\right)
$$

uniformly for $x \in \mathbb{R}$ and $t_{n} \in[0, T]$.

Proof Let $p(x, t)=\partial_{t} q(x, t)$. A straightforward application of Lemma 2.2 gives

$$
-h_{x}^{-\alpha} \delta_{x}^{\alpha} p(x, t)=\partial_{x}^{\alpha} p(x, t)+\mathcal{O}\left(h_{x}^{2}\right),
$$

or

$$
-h_{x}^{-\alpha} \delta_{x}^{\alpha} \partial_{t} q(x, t)=\partial_{x}^{\alpha} \partial_{t} q(x, t)+\mathcal{O}\left(h_{x}^{2}\right) .
$$

Let $t=t_{n-1}+2 \tau \theta$ in A.4. Integrating the result from 0 to 1 for $\theta$, we have

$$
-h_{x}^{-\alpha} \delta_{x}^{\alpha} \int_{0}^{1} \partial_{t} q\left(x, t_{n-1}+2 \tau \theta\right) \mathrm{d} \theta=\partial_{x}^{\alpha} \int_{0}^{1} \partial_{t} q\left(x, t_{n-1}+2 \theta \tau\right) \mathrm{d} \theta+\int_{0}^{1} \mathcal{O}\left(h_{x}^{2}\right) \mathrm{d} \theta .
$$

Notice that

$$
\int_{0}^{1} \partial_{t} q\left(x, t_{n-1}+2 \tau \theta\right) \mathrm{d} \theta=\Delta_{t} q\left(x, t_{n}\right)
$$

such that A.5 becomes

$$
-h_{x}^{-\alpha} \delta_{x}^{\alpha} \Delta_{t} q\left(x, t_{n}\right)=\partial_{x}^{\alpha} \Delta_{t} q\left(x, t_{n}\right)+\mathcal{O}\left(h_{x}^{2}\right),
$$

which implies A.3. 
Next, we derive the local truncation errors $R_{i j}^{n}$ in 3.11) and $\Delta_{t} R_{i j}^{n}$ in (3.12). Denote

$$
\begin{aligned}
& \left(R^{(1)}\right)_{i j}^{n}=\frac{\tau^{2}}{4} \int_{0}^{1}\left[\partial_{t t t} u\left(x_{i j}, t_{n}+s \tau\right)+\partial_{t t t} u\left(x_{i j}, t_{n}-s \tau\right)\right](1-s)^{2} d s \\
& \left(R^{(2)}\right)_{i j}^{n}=\frac{\tau^{2}}{2} \int_{0}^{1}\left[\partial_{t t} u\left(x_{i j}, t_{n}+s \tau\right)+\partial_{t t} u\left(x_{i j}, t_{n}-s \tau\right)\right](1-s) d s .
\end{aligned}
$$

Using A.1 and A.2 , we have

$$
\begin{aligned}
& \Delta_{t} U_{i j}^{n}-\partial_{t} u\left(x_{i}, y_{j}, t_{n}\right)=\left(R^{(1)}\right)_{i j}^{n}, \\
& \left|U_{i j}^{n}\right|^{2} U_{i j}^{\bar{n}}-\left|u\left(x_{i}, y_{j}, t_{n}\right)\right| u\left(x_{i}, y_{j}, t_{n}\right)=\left|U_{i j}^{n}\right|^{2} \cdot\left(R^{(2)}\right)_{i j}^{n},
\end{aligned}
$$

Using A.2 in Lemma A.7 and Lemma 2.2 we have

$$
\begin{aligned}
\delta_{x}^{\alpha} U_{i j}^{\bar{n}}-\partial_{x}^{\alpha} u\left(x_{i}, y_{j}, t_{n}\right) & =\partial_{x}^{\alpha}\left(R^{(2)}\right)_{i j}^{n}+\mathcal{O}\left(h_{x}^{2}\right), \\
\delta_{y}^{\beta} U_{i j}^{\bar{n}}-\partial_{y}^{\beta} u\left(x_{i}, y_{j}, t_{n}\right) & =\partial_{y}^{\beta}\left(R^{(2)}\right)_{i j}^{n}+\mathcal{O}\left(h_{y}^{2}\right) .
\end{aligned}
$$

Subtracting 3.10 from (3.19) and noticing A.6 A.9 we have

$$
R_{i j}^{n}=\left(R^{(1)}\right)_{i j}^{n}-(\nu+\mathbf{i} \eta)\left(\left(\partial_{x}^{\alpha}+\partial_{y}^{\beta}\right)\left(R^{(2)}\right)_{i j}^{n}\right)+(\kappa+\mathbf{i} \zeta)\left|U_{i j}^{n}\right|^{2} \cdot\left(R^{(2)}\right)_{i j}^{n}-\gamma\left(R^{(2)}\right)_{i j}^{n}+\mathcal{O}\left(h_{x}^{2}+h_{y}^{2}\right)
$$

Thus, the local truncation errors $R_{i j}^{n}$ in 3.11 holds. Furthermore, we have

$$
\begin{aligned}
\Delta_{t}\left(R^{(1)}\right)_{i j}^{n}= & \frac{1}{2 \tau}\left(\left(R^{(1)}\right)_{i j}^{n+1}-\left(R^{(1)}\right)_{i j}^{n-1}\right) \\
= & \frac{\tau}{8} \int_{0}^{1}\left[\partial_{t t t} u\left(x_{i}, y_{j}, t_{n+1}+s \tau\right)+\partial_{t t t} u\left(x_{i}, y_{j}, t_{n+1}-s \tau\right)\right](1-s)^{2} d s \\
& -\frac{\tau}{8} \int_{0}^{1}\left[\partial_{t t t} u\left(x_{i}, y_{j}, t_{n-1}+s \tau\right)+\partial_{t t t} u\left(x_{i}, y_{j}, t_{n-1}-s \tau\right)\right](1-s)^{2} d s \\
= & \frac{\tau}{8} \int_{0}^{1}\left[\partial_{t t t} u\left(x_{i}, y_{j}, t_{n+1}+s \tau\right)-\partial_{t t t} u\left(x_{i}, y_{j}, t_{n-1}+s \tau\right)\right](1-s)^{2} d s \\
& +\frac{\tau}{8} \int_{0}^{1}\left[\partial_{t t t} u\left(x_{i}, y_{j}, t_{n+1}-s \tau\right)-\partial_{t t t} u\left(x_{i}, y_{j}, t_{n-1}-s \tau\right)\right](1-s)^{2} d s \\
= & \frac{\tau^{2}}{4} \int_{0}^{1} \int_{0}^{1}\left[\partial_{t t t t} u\left(x_{i}, y_{j}, t_{n-1}+(2 \mu+s) \tau\right)+\partial_{t t t t} u\left(x_{i}, y_{j}, t_{n-1}+(2 \mu-s) \tau\right)\right](1-s)^{2} d \mu d s .
\end{aligned}
$$

Therefore,

$$
\left\|\Delta_{t}\left(R^{(1)}\right)^{n}\right\| \leqslant c \tau^{2},
$$

where $c$ is a positive constant. Similarly, we have

$$
\left\|\Delta_{t}\left(R^{(2)}\right)^{n}\right\| \leqslant c \tau^{2}, \quad\left\|\Delta_{t}\left(U^{n}\left(R^{2}\right)^{n}\right)\right\| \leqslant c \tau^{2} .
$$

Combining A.3 in Lemma A.8 and A.10, we have

$$
\left\|\Delta_{t}\left(\partial_{x}^{\alpha}\left(R^{(2)}\right)^{n}\right)\right\|=\mathcal{O}\left(\tau^{2}+h_{x}^{2}\right), \quad\left\|\Delta_{t}\left(\partial_{y}^{\beta}\left(R^{2}\right)^{n}\right)\right\|=\mathcal{O}\left(\tau^{2}+h_{y}^{2}\right)
$$

Thus, the local truncation errors $\Delta_{t} R_{i j}^{n}$ in 3.12 holds. 


\section{References}

1. Akhmediev, N.N., Ankiewicz, A., Soto-Crespo, J.M.: Multisoliton solutions of the complex GinzburgLandau equation. Phys. Rev. Lett. 79(21), 4047-4051 (1997)

2. Aranson, I.S., Kramer, L.: The world of the complex Ginzburg-Landau equation. Rev. Modern Phys. 74, 99-143 (2002)

3. Arshed, S.: Soliton solutions of fractional complex Ginzburg-Landau equation with Kerr law and non-Kerr law media. Optik, 160, 322-332 (2018)

4. Bao, W., Tang, Q.: Numerical study of quantized vortex interaction in the Ginzburg-Landau equation on bounded domains. Commun. Comput. Phys. 14(3), 819-850 (2013)

5. Bartels, S.: A posteriori error analysis for time-dependent Ginzburg-Landau type equations. Numer. Math. 99, 557-583 (2005)

6. Çelik, C., Duman, M.: Crank-Nicolson method for the fractional diffusion equation with the Riesz fractional derivative. J. Comput. Phys. 231, 1743-1750 (2012)

7. Defterli, O., D'Elia, M., Du, Q., Gunzburger, M., Lehoucq, R., Meerschaert, M.M.: Fractional diffusion on bounded domains. Fract. Calc. Appl. Anal. 18, 342-360 (2015)

8. Degong, P., Jin, S., Tang, M.: On the time splitting spectral method for the complex Ginzburg-Landau equation in the large time and space scale limit. SIAM J. Sci. Comput. 30(5), 2466-2487 (2008)

9. Du, Q., Gunzburger, M., Lehoucq, R.B., Zhou, K.: Analysis and approximation of nonlocal diffusion problems with volume constraints. SIAM Rev. 54, 667-696 (2012)

10. Du, Q., Gnnzburger, M., Peterson, J.: Finite element approximation of a periodic Ginzburg-Landau model for type-II superconductors. Numer. Math. 64, 85-114 (1993)

11. Feynman, R.P., Hibbs, A.R.: Quantum Mechanics and Path Integrals. McGraw-Hill, New York, (1965)

12. Gorenflo, R., Mainardi, F.: Random walk models for space-fractional diffusion processes. Fract. Calc. Appl. Anal. 1(2), 167-191 (1998)

13. Guo, B., Huo, Z.: Well-posedness for the nonlinear fractional Schrödinger equation and inviscid limit behavior of solution for the fractional Ginzburg-Landau equation. Fract. Calc. Appl. Anal. 16(1), 226-242 (2013)

14. Hao, Z.P., Sun, Z.Z.: A linearized high-order difference scheme for the fractional Ginzburg-Landau equation. Numer. Meth. Part. D. E. 33(1), 105-124 (2017)

15. He, D., Pan, K.: An unconditionally stable linearized difference scheme for the fractional GinzburgLandau equation. Numer. Algorithms 79(3), 899-925 (2018)

16. Hu, H.L., Jin, X., He, D., Pan, K., Zhang, Q.: A conservative difference scheme with optimal pointwise error estimates for two-dimensional space fractional nonlinear Schrödinger equations. ArXiv: https://arxiv.org/abs/1910.08311

17. Kirkpatrick, K., Lenzmann, E., Staffilani, G.: On the continuum limit for discrete NLS with long-range lattice interactions. Commun. Math. Phys. 317, 563-591 (2013)

18. Kitzhofer, G., Koch, O., Weinmüller, E.B.: Pathfollowing for essentially singular boundary value problems with application to the complex Ginzburg-Landau equation. BIT Numer. Math. 49, 141-160 (2009)

19. Laskin, N.: Fractional quantum mechanics. Phys. Rev. E 62, 3135-3145 (2000)

20. Laskin, N.: Fractional quantum mechanics and Lévy path integrals. Phys. Lett. A 268, $298-305$ (2000)

21. Li, M., Huang, C.: An efficient difference scheme for the coupled nonlinear fractional Ginzburg-Landau equations with the fractional Laplacian. Numer. Meth. Part. D. E. 35(1), 394-421 (2019)

22. Li, M., Huang, C., Wang, N.: Galerkin element method for the nonlinear fractional Ginzburg-Landau equation. Appl. Numer. Math. 118, 131-149 (2017)

23. Lu, H., Bates, P.W., Lü, S.J., Zhang, M.J.: Dynamics of the 3-D fractional complex Ginzburg-Landau equation. J. Differ. Equations 259, 5276-5301 (2015)

24. Lu, H., Lü, S.J., Zhang, M.J.: Fourier spectral approximations to the dynamics of 3D fractional complex Ginzburg-Landau equation. Discrete Cont. Dyn. S.-A 37(5), 2539-2564 (2017)

25. Millot, V., Sire, Y.: On a fractional Ginzburg-Landau equation and 1/2-Harmonic maps into spheres. Arch. Ration. Mech. Anal. 215, 125-210 (2015)

26. Mohebbi, A.: Fast and high-order numerical algorithms for the solution of multidimensional nonlinear fractional Ginzburg-Landau equation. Eur. Phys. J. Plus 133(2), 67 (2018)

27. $\mathrm{Pu}, \mathrm{X}$., Guo, B.: Well-posedness and dynamics for the fractional Ginzburg-Landau equation. Appl. Anal. 92(2), 31-33 (2013)

28. Shu, J., Li, P., Zhang, J., Liao, O.: Random attractors for the stochastic coupled fractional GinzburgLandau equation with additive noise. J. Math. Phys. 56, 102702 (2015)

29. Sun, Z.Z.: A second-order accurate linearized difference scheme for the two-dimensional Cahn-Hilliard equation. Math. Comp. 64, 1463-1471 (1995) 
30. Takac, P., Jungel, A.: A nonstiff Euler discretization of the complex Ginzburg-Landau equation in one space dimension, SIAM J. Numer. Anal. 38(1), 292-328 (2000)

31. Sakaguchi, H., Malomed, B.A.: Stable solitons in coupled Ginzburg-Landau equations describing BoseEinstein condensates and nonlinear optical waveguides and cavities. Physica D 183, $282-292$ (2003)

32. Tarasov, V., Zaslavsky, G.: Fractional dynamics of coupled oscillators with long-range interaction. Chaos 16, $023110(2006)$

33. Tarasov, V., Zaslavsky, G.: Fractional Ginzburg-Landau equation for fractal media. Physica A 354, 249-261 (2005)

34. Wang, P., Huang, C.: An efficient fourth-order in space difference scheme for the nonlinear fractional Ginzburg-Landau equation. BIT. 58(3), 783-805 (2018)

35. Wang, P., Huang, C.: Split-step alternating direction implicit difference scheme for the fractional Schrödinger equation in two dimensions. Comput. Math. Appl. 71(5), 1114-1128 (2016)

36. Wu, Y.S.: Multiparticle quantum mechanics obeying fractional statistics. Phys. Rev. Lett. 53, 111-114 (1984)

37. Zeng, F., Liu, F., Li, C., Burrage, K., Turner, I., Anh, V.: A Crank-Nicolson ADI spectral method for a two-dimensional Riesz space fractional nonlinear reaction-diffusion equation. SIAM J. Numer. Anal. 52(6), 2599-2622 (2014)

38. Zhang, M., Zhang, G.F., Liao, L.D.: Fast iterative solvers and simulation for the space fractional Ginzburg-Landau equations Ginzburg-Landau equations. Comput. Math. Appl. 78(5), 1793-1800 (2019)

39. Zhang, Q., Pan, K., Lin, X., Ren, Y.: Linearized ADI schemes for two-dimensional space-fractional nonlinear Ginzburg-Landau equation. Comput. Math. Appl. 80, 1201-1220 (2020)

40. Zhang, Q., Zhang, L., Sun, H.: A three-level finite difference method with preconditioning technique for two-dimensional nonlinear fractional complex Ginzburg-Landau equations. Revised.

41. Zhao, X., Sun, Z., Hao, Z.: A fourth-order compact ADI scheme for two-dimensional nonlinear space fractional Schrödinger equation. SIAM J. Sci. Comput. 36(6), 2865-2886 (2014) 